\title{
Renal tubule Cpt1a overexpression protects from kidney fibrosis by restoring mitochondrial homeostasis
}

\author{
Verónica Miguel, ${ }^{1}$ Jessica Tituaña, ${ }^{1}$ J. Ignacio Herrero, ${ }^{1}$ Laura Herrero, ${ }^{2,3}$ Dolors Serra, ${ }^{2,3}$ Paula Cuevas, ${ }^{4}$ Coral Barbas, ${ }^{4}$ \\ Diego Rodríguez Puyol, ${ }^{5}$ Laura Márquez-Expósito, ${ }^{6}$ Marta Ruiz-Ortega, ${ }^{6}$ Carolina Castillo, ${ }^{7}$ Xin Sheng, ${ }^{8}$ Katalin Susztak, ${ }^{8}$ \\ Miguel Ruiz-Canela, ${ }^{9,10}$ Jordi Salas-Salvadó, ${ }^{10,11}$ Miguel A. Martínez González, ${ }^{9}{ }^{90}$ Sagrario Ortega, ${ }^{12}$ Ricardo Ramos, ${ }^{13}$ \\ and Santiago Lamas'
}

\begin{abstract}
Program of Physiological and Pathological Processes, Centro de Biologia Molecular "Severo Ochoa" (CBMSO) (CSIC-UAM), Madrid, Spain. 'Department of Biochemistry and Physiology, School of Pharmacy and Food Sciences, Institut de Biomedicina de la Universitat de Barcelona (IBUB), Universitat de Barcelona, Barcelona, Spain. ${ }^{3}$ Centro de Investigación Biomédica en Red de Fisiopatología de la Obesidad y la Nutrición (CIBEROBN), Instituto de Salud Carlos III, Madrid, Spain. ${ }^{4}$ Centre for Metabolomics and Bioanalysis (CEMBIO), Chemistry and Biochemistry Department, Pharmacy Faculty, Universidad San Pablo-CEU, Boadilla del Monte, Madrid, Spain. ${ }^{5}$ Department of Medicine and Medical Specialties, Research Foundation of the University Hospital "Principe de Asturias," IRYCIS, Alcalá University, Alcalá de Henares, Madrid, Spain. ${ }^{6}$ Cellular and Molecular Biology in Renal and Vascular Pathology Laboratory. Fundación Instituto de Investigación Sanitaria-Fundación Jiménez Díaz-Universidad Autónoma Madrid, Madrid, Spain. 'University Hospital “Principe de Asturias”, Alcalá de Henares, Madrid, Spain. ${ }^{8}$ Division of Nephrology, Department of Medicine, Department of Cenetics, Perelman School of Medicine, University of Pennsylvania, Philadelphia, Pennsylvania, USA. 'Pniversity of Navarra, Department of Preventive Medicine and Public Health, IdiSNA (Health Research Institute of Navarra), Pamplona, Spain. ${ }^{10}$ CIBER de Fisiopatología de la Obesidad y Nutrición (CIBEROBN), Instituto de Salud Carlos III, Madrid, Spain. "Human Nutrition Unit, Faculty of Medicine and Health Sciences, Pere Virgili Health Research Institute, Rovira i Virgili University, Reus, Spain. ${ }^{2}$ Transgenics Unit, Spanish National Cancer Research Centre (CNIO), Madrid, Spain. ${ }^{13}$ Cenomic Facility, Parque Cientifico de Madrid, Madrid, Spain.
\end{abstract}

\begin{abstract}
Chronic kidney disease (CKD) remains a major epidemiological, clinical, and biomedical challenge. During CKD, renal tubular epithelial cells (TECs) present a persistent inflammatory and profibrotic response. Fatty acid oxidation (FAO), the main source of energy for TECs, is reduced in kidney fibrosis and contributes to its pathogenesis. To determine whether gain of function in FAO (FAO-GOF) could protect from fibrosis, we generated a conditional transgenic mouse model with overexpression of the fatty acid shuttling enzyme carnitine palmitoyl-transferase 1A (CPT1A) in TECs. Cpt1a-knockin (CPT1A-KI) mice subjected to 3 models of renal fibrosis (unilateral ureteral obstruction, folic acid nephropathy [FAN], and adenine-induced nephrotoxicity) exhibited decreased expression of fibrotic markers, a blunted proinflammatory response, and reduced epithelial cell damage and macrophage influx. Protection from fibrosis was also observed when Cpt1a overexpression was induced after FAN. FAO-GOF restored oxidative metabolism and mitochondrial number and enhanced bioenergetics, increasing palmitate oxidation and ATP levels, changes that were also recapitulated in TECs exposed to profibrotic stimuli. Studies in patients showed decreased CPT1 levels and increased accumulation of short- and middlechain acylcarnitines, reflecting impaired FAO in human CKD. We propose that strategies based on FAO-COF may constitute powerful alternatives to combat fibrosis inherent to CKD.
\end{abstract}

\section{Introduction}

Organ fibrosis constitutes a significantly prevalent pathological entity associated with high morbidity and mortality and presents a major biomedical challenge. Although fibrosis may affect any tissue of the human body, its presence in the kidney generally indicates unrelenting progression to chronic renal failure, a condition associated with reduced expectancy and quality of life. Hence, fibrosis is a convergent pathway for prevalent pathologies underlying chronic kidney disease (CKD), such as diabetes, hypertension, and nephrosclerosis (1). Beyond proper blood pressure and glycemic controls, therapeutic options to revert or deter the progression of fibrosis are very limited. In the last few years, the

Conflict of interest: The authors have declared that no conflict of interest exists. Copyright: () 2021, American Society for Clinical Investigation.

Submitted: May 27, 2020; Accepted: January 13, 2021; Published: March 1, 2021

Reference information: J Clin Invest. 2021;131(5):e140695.

https://doi.org/10.1172/JCl140695. understanding of major metabolic disturbances coexisting with kidney fibrosis have shed new light on the pathogenesis of fibrosis progression (2). Among these, a drastic reduction in fatty acid oxidation (FAO) appears to be critical for the global energy failure occurring in the tubulo-interstitial compartment, contributing to immune cell infiltration and kidney fibrosis $(3,4)$. Here, we tested whether specific metabolic gain of function in FAO (FAO-GOF) in renal tubules was necessary and sufficient to counteract cellular and molecular changes associated with kidney fibrosis. Carnitine palmitoyl transferase $1 \mathrm{~A}(\mathrm{CPT} 1 \mathrm{~A})$ is a rate-limiting and targetable enzyme in this pathway. We found that conditional overexpression of CPT1A resulted in significant mitigation of fibrosis and improvement of renal function in 3 experimental models. This was related to enhanced mitochondrial mass, repaired architecture, and bioenergetic recovery. The overexpression of CPT1A was also associated with a reduced inflammatory pattern and abrogation of TGF- $\beta$-induced epithelial cell damage. In the folic acid nephropathy (FAN) model, pathological changes were markedly 
reverted when FAO-GOF was reinstated. Moreover, studies in patients with chronic renal failure showed that a reduction in the levels of CPT1A correlated with the degree of fibrosis. In addition, in a large cohort of diabetic patients with $\mathrm{CKD}$, a specific profile of increased plasma acylcarnitines was found, reinforcing the critical metabolic derangement of FAO associated with CKD.

\section{Results}

Overexpression of tubular CPT1A results in protection against kidney fibrosis. To test the in vivo relevance of CPT1A and FAO for renal fibrosis, a mouse model with conditional, inducible expression of Cpt1a was engineered as described in Methods. To ensure renal epithelial tissue specificity, these animals were crossed with mice expressing an optimized reverse tetracycline-controlled transactivator (rtTA2s-M2) driven by the paired box 8 (Pax8) promoter, hereafter termed Pax8-rtTA mice. Extrarenal expression of Pax8 only occurs at the level of the thyroid without reported off-target effects (5). This results in tissue-specific expression of the Cpt1a gene bearing the tetracycline-responsive promoter element in the proximal and distal tubules and collecting duct, which is evident 2 weeks after doxycycline administration (Figure 1, A and B and Supplemental Figure 1, A and B; supplemental material available online with this article; https://doi.org/10.1172/JCI140695DS1). We next characterized the renal-specific overexpression of CPT1A in this newly generated genetic mouse model based on the doxycycline-inducible transgenic system Tet-On. CPT1A-KI mice presented a 10-fold increase in the CPT1A mRNA level in whole kidney tissue compared with WT mice (Figure1C). This was accompanied by a marked augmentation of CPT1A protein expression in the kidney. Of note, no differences were observed when the liver tissue was analyzed (Figure 1, D and E). Tubules were labeled by lotus tetragonolobus lectin, a marker for proximal tubules. Consistently, induced expression of CPT1A and GFP proteins was colocalized in tubule segments as evaluated by immunohistochemistry (Figure 1F). Importantly, overexpressed CPT1A presented a mitochondrial localization pattern, which was observed by double immunostaining by using the ATP synthase beta-subunit ( $\beta F 1)$ as a marker (Figure $1 G)$. To assess the magnitude of CPT1A overexpression on FAO, we analyzed the capacity of renal tissue to oxidize radiolabeled palmitate. Functionally, the increase in CPT1A protein in the renal epithelium increased the capacity of kidney tissue to oxidize ${ }^{14} \mathrm{C}$-palmitate as reflected in the levels of both ${ }^{14} \mathrm{C}$-palmitate-derived ${ }^{14} \mathrm{CO}_{2}$ as well as in ${ }^{14} \mathrm{C}$-palmitate-derived acid-soluble products (Figure $1 \mathrm{H}$ ).

To evaluate the effect of the Cpt1a-knockin (CPT1A-KI) strategy on renal damage, we first used the FAN injury model (Supplemental Figure 1C, upper panel). Although we found no significant differences in the degree of tubular atrophy and dilatation (Supplemental Figure 2, A-C), fibrosis was markedly ameliorated in Pax8-rtTA ${ }^{\mathrm{tg} / 0}$ tetO-Cpt1a ${ }^{\mathrm{tg} / 0}$ mice compared with WT mice (Figure 2A, left panel) and a significant reduction in the proportion of fibrosis as quantified by Sirius red was observed in the CPT1A-KI model (Figure 2A, right panel). Renal function was reduced in FAN-treated WT animals, as reflected by the increase in blood urea nitrogen (BUN) and creatinine, but this effect was blunted in mice overexpressing CPT1A (Figure 2, B and C). The protein expression of the classic profibrotic markers fibronec- tin (FN) and smooth muscle actin (aSMA) was also significantly reduced in the CPT1A-KI mice (Figure 2, D and E). To confirm the beneficial effect of FAO-GOF in kidney fibrosis, the latter was assessed by performing unilateral ureteral obstruction (UUO) for 3 and 7 days in mice overexpressing CPT1A in tubular epithelial cells (TECs) (Supplemental Figure 1D). Obstructed kidneys from WT animals showed significant tubulo-interstitial architectural and histological changes 7 days after UUO, whereas the extent of tubular atrophy and dilatation was markedly reduced in kidneys with increased levels of CPT1A (Supplemental Figure 3A, left panel). Evaluation of renal lesions by light microscopy showed increased collagen deposition in the interstitial area after 7 days of the procedure in the obstructed kidneys compared with the contralateral ones in WT mice. A significant protective effect of CPT1A overexpression was observed by a reduction of $20 \%-40 \%$ in collagen deposition (Supplemental Figure 3A, right panel). As expected, circulating levels of BUN and creatinine were not different between WT and CPT1A-KI mice after 7 days of UUO because of the remaining functional kidney (Supplemental Figure 3, B and C). A less dramatic but still significant decrease in FN and $\alpha \mathrm{SMA}$ was also observed (Supplemental Figure 3, D and E).

To understand the mechanisms underlying the protective action of CPT1A on renal fibrosis, we performed expression analysis of whole kidney tissue from WT or CPT1A-KI mice subjected to FAN or 7 days of UUO. Expression of genes related to critical cellular functions for the initiation and perpetuation of tubular dysfunction and chronic tissue damage was analyzed by using specific TaqMan probes. A reduced expression for this subset of genes was observed in the FAN model. Quantitative real-time PCRbased (qRT-PCR-based) quantification displayed lower expression of epithelial injury (Havcr1-KIM-1) and fibrotic markers (collagens) in kidneys from CPT1A-KI mice (Figure 2F). These data were confirmed by mRNA quantitative analysis using SYBR green (Supplemental Figure 4A). A similar effect was observed in the UUO model (Supplemental Figure $3 \mathrm{~F}$ and Supplemental Figure $4 \mathrm{~B})$. These observations were also confirmed in the CKD mouse model of adenine-induced nephrotoxicity (ADN, Supplemental Figure 1E). CPT1A-KI mice showed a reduction in the expression of the crucial genes Col1a1, Fn1, and TGF- $\beta$ involved in these cellular mechanisms related to kidney fibrosis (Supplemental Figure 5 , A and B) as well as the same trend in the injury marker KIM-1 (Supplemental Figure 5, C and D). Data collected from the 3 models of CKD in the CPT1A-KI mice strongly indicate that CPT1A is an enzyme that by itself has a crucial impact on the outcome of fibrosis, most likely because of its critical function in the facilitation of FAO.

CPT1A overexpression prevents mitochondrial dysfunction and restores $F A O$ in the fibrotic kidney. Renal mitochondrial abnormalities and dysfunction are common features in the pathogenesis of different forms of renal disease (6). Cellular pathways promoting kidney damage can compromise mitochondrial homeostasis, reflected in increased oxidative stress, apoptosis, microvascular loss, and fibrosis, all of which contribute to renal function deterioration (7). Thus, we evaluated morphological alterations of mitochondria in cortical proximal tubules by transmission electron microscopy. Cells from tubular segments of healthy kidneys presented regular apical microvilli, intact basement membrane, and 
A

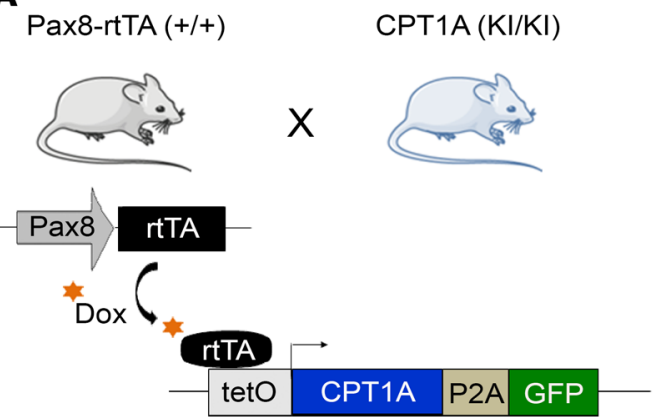

B

Pax8-rtTA tetO-Cpt $1 \mathrm{a}^{\mathrm{tg} / 0}$ tetO-Cpt1 $\mathrm{a}^{\mathrm{tg} / 0}$ tetO-Cpt $1 \mathrm{a}^{\mathrm{O} / 0}$

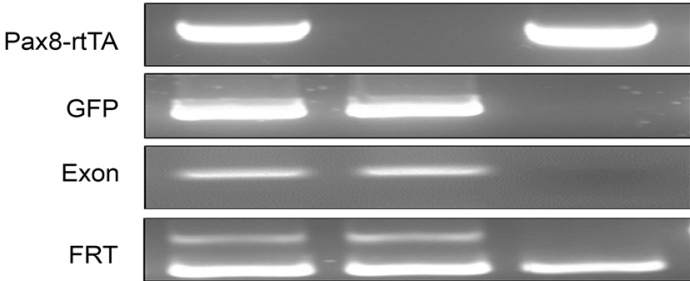

C

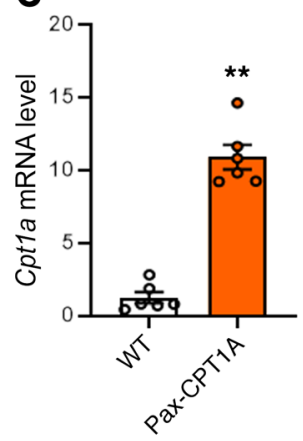

D

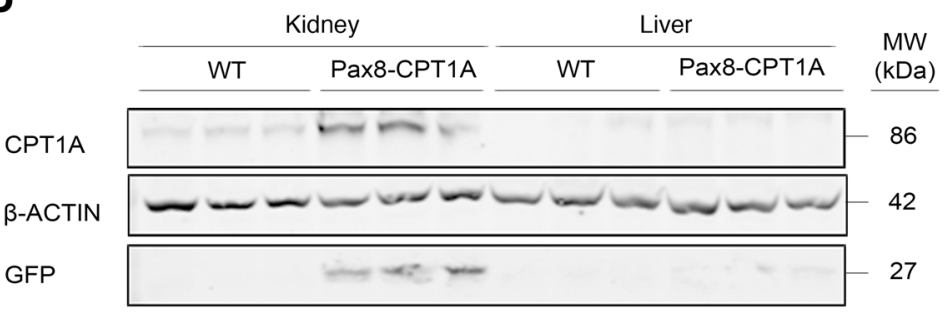

E

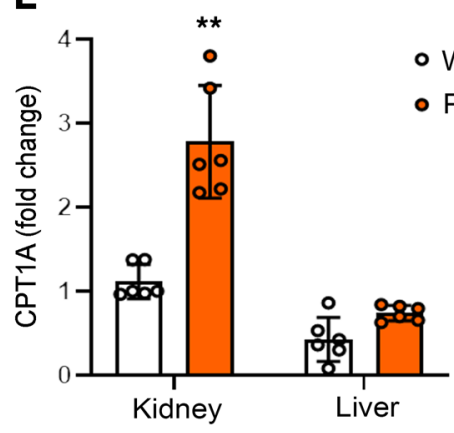

G

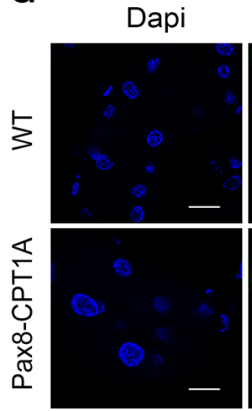

$\beta F 1$

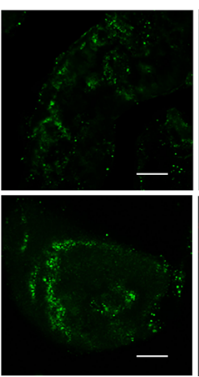

CPT1A

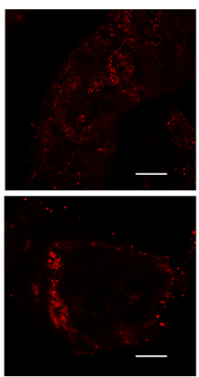

$\mathbf{F}$

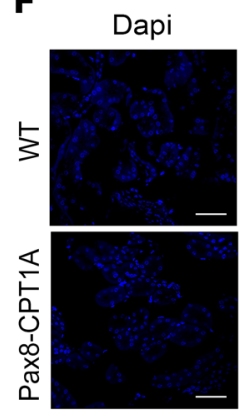

GFP

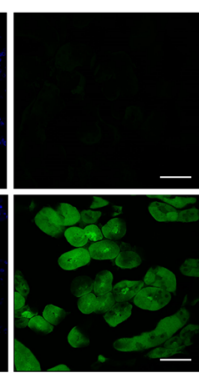

LTL
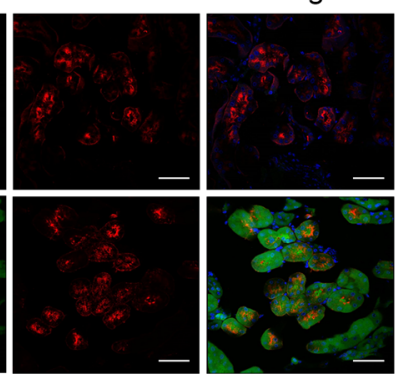

H
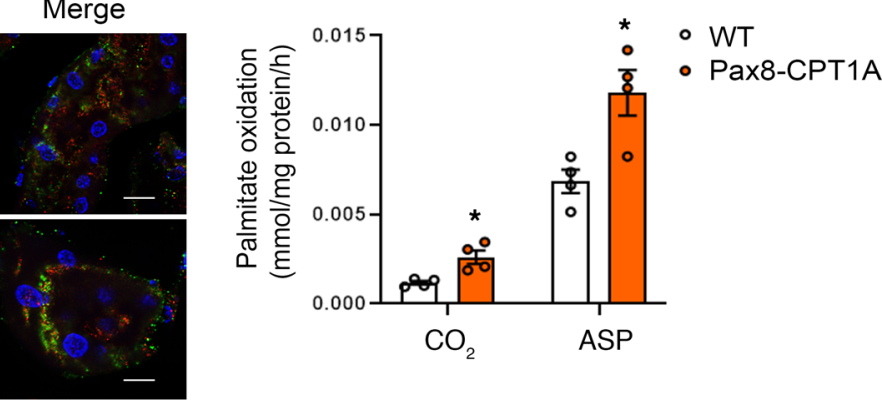

Figure 1. Characterization of doxycycline-inducible Cpt1a gene overexpression in the transgenic mouse model for the inducible Cpt1a gene in renal epithelial cells. (A) Schematic depicting the strategy to generate mice with inducible renal tubular epithelial cell-specific overexpression of CPT1A. (B) PCR analysis of offspring genotypes from these crosses. Genomic DNA analysis by PCR for the Pax8-rtTA allele generates a 595-bp band (Supplemental Table 1). The GFP, exon, and FRT PCRs are described in Supplemental Figure 1A. (C) mRNA levels of the Cpt1a gene were determined by qRT-PCR in total kidney tissue of mice treated with doxycycline for 3 weeks. Data represent the mean \pm SEM $\left(n=6\right.$ mice). ${ }^{* *} P<0.05$ compared with kidneys from WT mice. (D) Immunoblots depicting protein levels of CPT1A and GFP in kidneys and livers of 3 individual mice per group. $\beta$-actin was used for normalization purposes. (E) Bar graphs represent the mean \pm SEM of fold changes corresponding to densitometric analyses $\left(n=6\right.$ mice). ${ }^{* *} P<0.01$ compared with kidneys from WT mice. (F) Representative images of double immunofluorescence staining with the proximal tubular marker lotus tetragonolobus lectin (LTL), GFP, DAPI (nuclei), and merge of all 3. (C) Staining with DAPI (nuclei), CPT1A, and the mitochondrial marker ATP synthase beta-subunit ( $\beta F 1$ ). All panels show immunofluorescence images of kidneys from WT and Pax8-CPT1A mice after doxycycline administration. Scale bar: $100 \mu \mathrm{m}$ (F and $\mathbf{G})$. (H) Radiolabeled palmitate-derived $\mathrm{CO}_{2}$ and acid-soluble products (ASP) were determined after incubation of ${ }^{14} \mathrm{C}$-palmitate with kidney tissue from WT or Pax8-CPT1A mice after doxycycline treatment. Bar graphs represent the mean \pm SEM $\left(n=4\right.$ mice). ${ }^{*} P<0.05$ compared with kidneys from WT mice. Statistical significance between 2 independent groups was determined using nonparametric 2-tailed Mann-Whitney $U$ test; more than 2 groups were compared with Kruskal-Wallis test. 
A

A $\frac{\text { Control }}{W T} \underset{\text { Pax8-CPT1A }}{W} \frac{\text { WT }}{\text { Pax8-CPT1A }}+$ DOX

$\underset{\infty}{\varpi}$
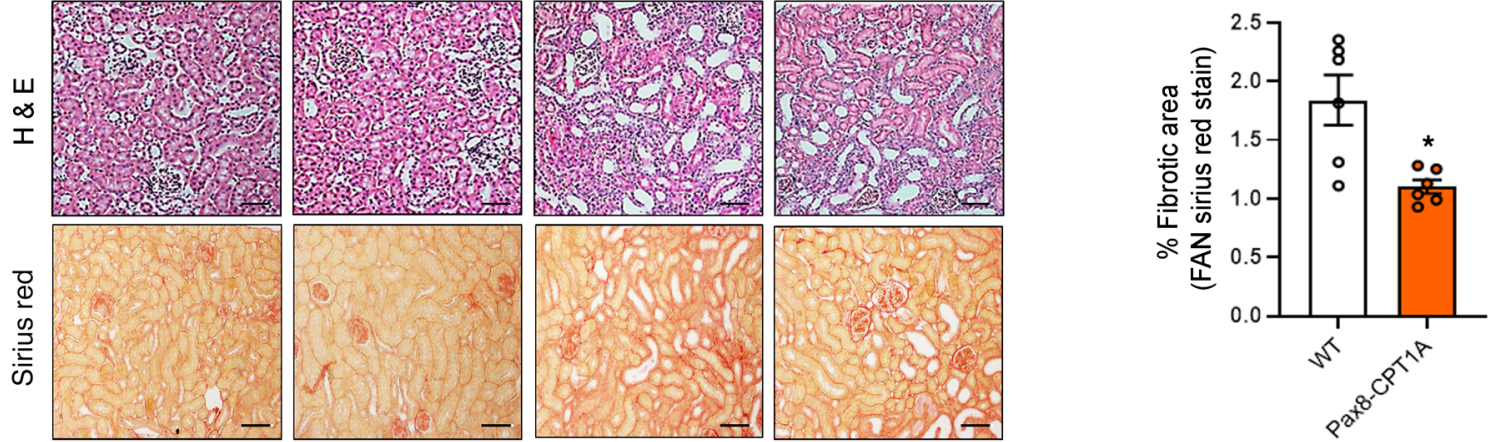

B

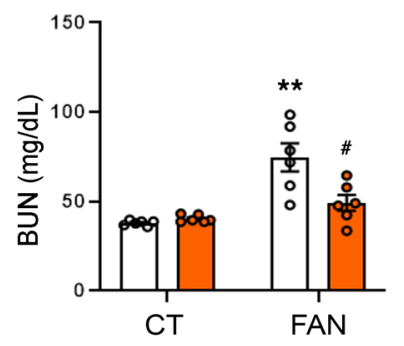

C

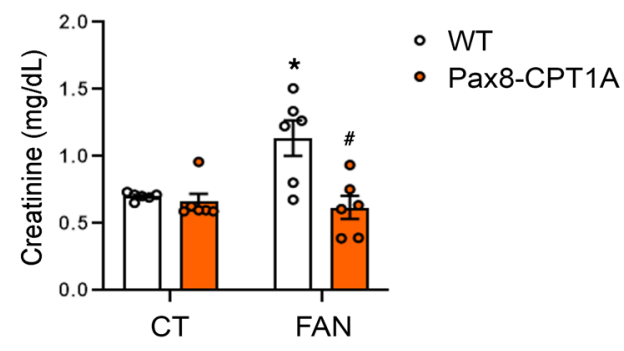

D
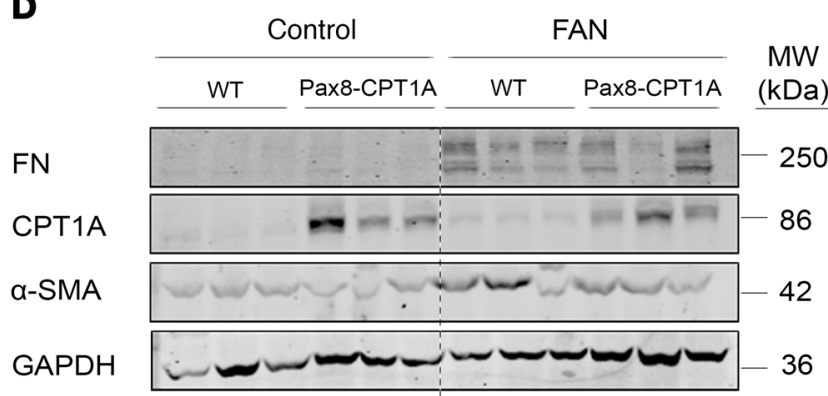

GFP

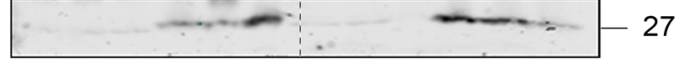

$\mathbf{F}$

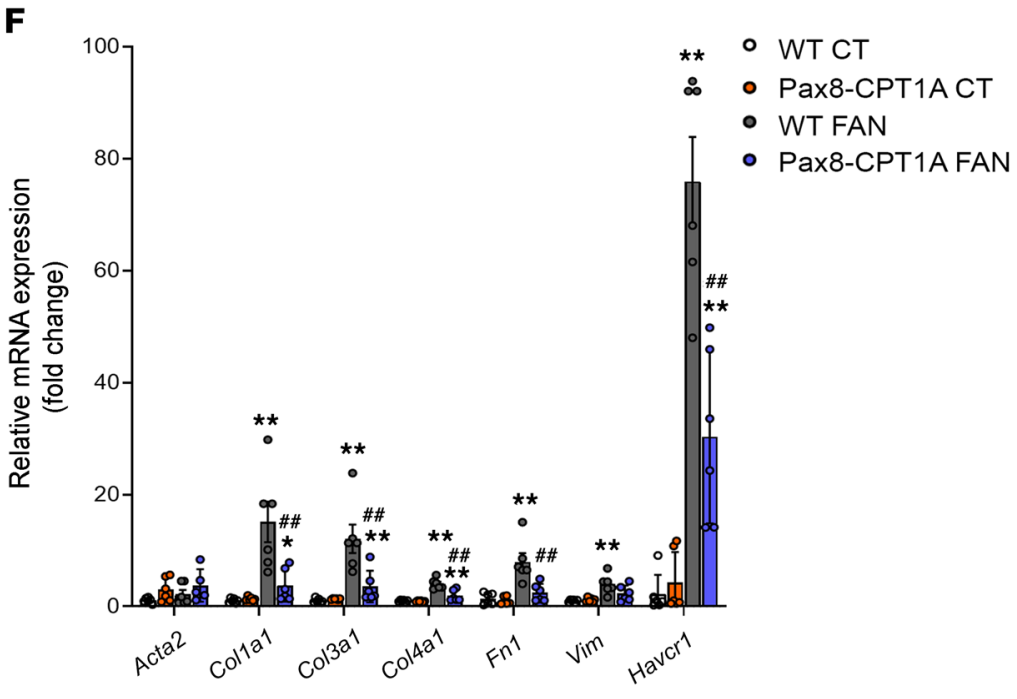

\section{E}

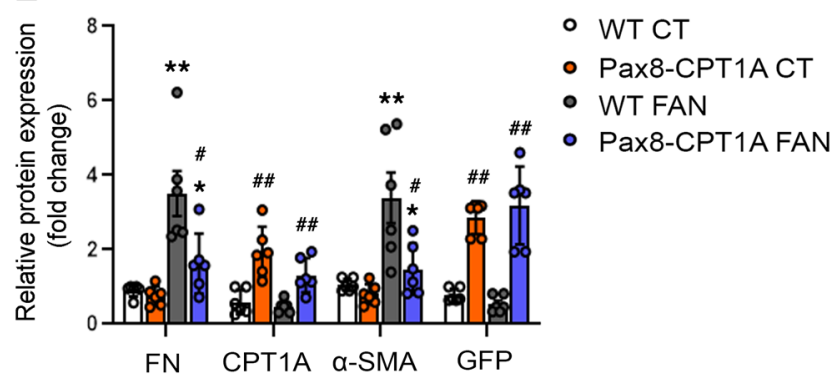


Figure 2. CPT1A overexpression prevents FAN-associated kidney function deterioration and experimental renal fibrosis. (A) Representative microphotographs from 1 mouse per group of H\&E (upper panels) and Sirius red (lower panels) staining of kidneys from WT and Pax8-CPT1A mice subjected to FAN after doxycycline treatment (Dox). Scale bars: $50 \mu \mathrm{m}$. Quantification of Sirius red staining represents the mean \pm SEM, $n=6$ mice. ${ }^{*} P<0.05$ compared with FAN/C kidneys in WT mice, respectively. (B and C) Serum blood urea nitrogen (BUN) (B) and serum creatinine (C) levels of WT and Pax8-CPT1A mice subjected to FAN after doxycycline treatment. Data represent the mean \pm SEM $\left(n=6\right.$ mice). ${ }^{*} P$ $<0.05$, ${ }^{* *} P<0.01$ compared with respective experimental control (CT) condition; ${ }^{*} P<0.05$ compared with WT mice with the same experimental condition. (D) Immunoblots depicting fibronectin (FN), carnitine palmitoyltransferase 1A (CPT1A), GFP, and alpha-smooth muscle actin $(\alpha-S M A)$ protein levels in kidneys from control (CT) and FA-treated (FAN) WT and Pax8-CPT1A mice after doxycycline induction. (E) Bar graphs represent the mean \pm SEM of fold changes corresponding to densitometric analyses ( $n=6$ mice). (F) mRNA levels of fibrosis-associated genes were determined by qRT-PCR using TaqMan qPCR probes in kidneys from CT and FAN WT and Pax8-CPT1A mice after doxycycline induction. Bar graphs represent the mean \pm SEM of fold changes $(n=6$ mice). ( $\mathbf{E}$ and $\mathbf{F}){ }^{*} P<0.05,{ }^{*} P<0.01$ compared with their corresponding CT kidneys; ${ }^{\#} P<0.05,{ }^{\#} P<0.01$ compared with kidneys from WT mice with the same experimental condition. Statistical significance between 2 independent groups was determined using nonparametric 2-tailed Mann-Whitney $U$ test; more than 2 groups were compared with KruskalWallis test. For gene nomenclature, see Supplemental Table 4.

basal infoldings. Mitochondria were very abundant; most of them presented an elongated shape and were localized in the basolateral part of the cells. They displayed a well-defined arrangement of well-preserved mitochondrial cisternae with a homogeneous inner matrix. In contrast, in the fatty acid-treated mice group, many epithelial cells were detached from the tubular basement membrane and showed disrupted basal infoldings. Mitochondrial structure was lost, and mitochondria presented a fragmented, small, and round appearance. Interestingly, most of these morphological alterations in mitochondria as well as the reduction in mitochondrial mass induced by fatty acids were almost abrogated in renal epithelial cells overexpressing CPT1A (Figure 3A). CPT1A overexpression prevented the drop in mtDNA copy number in the 3-day UUO (Supplemental Figure 6A) and FAN (Figure 3B) models of kidney damage. As expected, defective FAO was observed in fibrotic kidneys from WT mice. However, in the 3-day UUO, FAN, and ADN models, CPT1A overexpression counteracted this impairment, maintaining an FAO rate comparable to healthy kidneys (Figure 3C and Supplemental Figure 6, B and D). Closely related to the improvement in FAO by CPT1A, ATP content in whole kidney tissue increased from 50 to $80 \mu \mathrm{M} / \mathrm{mg}$ protein after CPT1A overexpression. In the 3-day UUO, FAN, and ADN models, CPT1A overexpression rescued the drop in ATP levels (Figure 3D and Supplemental Figure 6, C and E). These results suggest that appropriate levels of CPT1A and metabolic function are necessary and sufficient to preserve adequate mitochondrial architecture and morphology.

CPT1A GOF results in enhanced FAO-associated respiration of renal TECs even at the expense of glycolysis and AMPK activation. TECs use glucose for anaerobic glycolysis. Metabolic alterations of these cells during kidney fibrosis not only involve a defect in FAO but also in glucose oxidation (3). We found that in the FAN model, there was a general downregulation trend in the expression of glycolysis-related genes, which was not recovered by CPT1A overexpression (Figure 3E). By contrast, FAN-induced repression in mRNA levels of the peroxisomal/mitochondrial function-related genes Acox1, Cpt2, Lrpprc, Sdha, and Tfam was prevented in kidneys from CPT1A-KI mice (Figure 3F). In the UUO model, we found that the levels of the majority of the analyzed regulators of glucose utilization were not altered in obstructed kidneys compared with contralateral ones. Only the increased expression of $L d h 1$ and $S l c 2 a 1$ genes induced by UUO was prevented by CPT1A overexpression (Supplemental Figure 6F). Similarly, CPT1A GOF did not induce a major shift toward the expression of peroxisome/ mitochondrial-related genes in the contralateral kidneys (Supplemental Figure 6G).

To gain insight about quantitative metabolic changes at the cellular level, we examined the oxygen consumption rate (OCR) and extracellular acidification rate (ECAR) of primary TECs isolated from the kidneys of CPT1A-KI mice (Figure 4A). We found that basal and maximum OCRs were markedly higher when palmitic acid was supplied to TECs, indicating that TECs efficiently metabolized palmitate. The increase in OCR was sensitive to the CPT1 inhibitor etomoxir, confirming its specificity (Figure 4B). FAO-associated OCR was also higher in primary kidney epithelial cells isolated from CPT1A-KI compared with the ones isolated from WT mice. Cells treated with TGF- $\beta 1$ had a lower baseline of oxygen consumption levels and showed a reduction in palmitate-induced elevation in OCR, indicating a low activity of fatty acid metabolism. CPT1A overexpression prevented TGF- $\beta 1-$ induced bioenergetic derangement (Figure 4C). Simultaneously, glycolytic function associated with palmitate consumption was determined by ECAR. Oligomycin-induced blockage of OXPHOS allows determination of maximum glycolytic capacity. We found that CPT1A overexpression promoted the inhibition of basal glycolytic function both in the presence and absence of TGF- $\beta 1$ (Figure 4D). The increase in ATP levels related to CPT1A overexpression was also preserved under treatment with TGF- $\beta 1$ (Figure $4 \mathrm{E}$ ).

To confirm the metabolic functional consequences of CPT1A overexpression in the human setting, we examined OCR and ECAR in the human tubular epithelial cell line, HKC-8. Adenoviruses carrying CPT1A (AdCPT1A) or adenovirus control (AdControl) as a negative control were used to infect HKC- 8 cells. CPT1A protein levels were 4 -fold higher in CPT1A-expressing $\mathrm{HKC}-8$ cells than in control cells (Supplemental Figure 7, A and B). Consistent with our observations in epithelial cells from murine kidneys overexpressing CPT1A, HKC-8 cells transduced with CPT1A exhibited reduced TGF- $\beta 1$-induced FAO inhibition (Supplemental Figure 7C) and suppression of glycolysis, as reflected by ECAR levels (Supplemental Figure 7D). As expected, we found that the FAO rate was 2-fold higher in AdCPT1A-expressing HKC-8 cells, as confirmed by measurement of ${ }^{14} \mathrm{C}$-palmitate-derived ${ }^{14} \mathrm{CO}_{2}$ (Supplemental Figure 7E). Consistently, CPT1A overexpression increased the fluorescence resonance energy transfer (FRET) signal of a specific ATP sensor and promoted a reduced decrement in the presence of TGF- $\beta 1$ (Supplemental Figure 7F).

Depressed carbohydrate, amino acid, and lipid oxidative pathways have been described in patients and in animal models of CKD, leading to energy deprivation (6). AMPK is a highly con- 
A

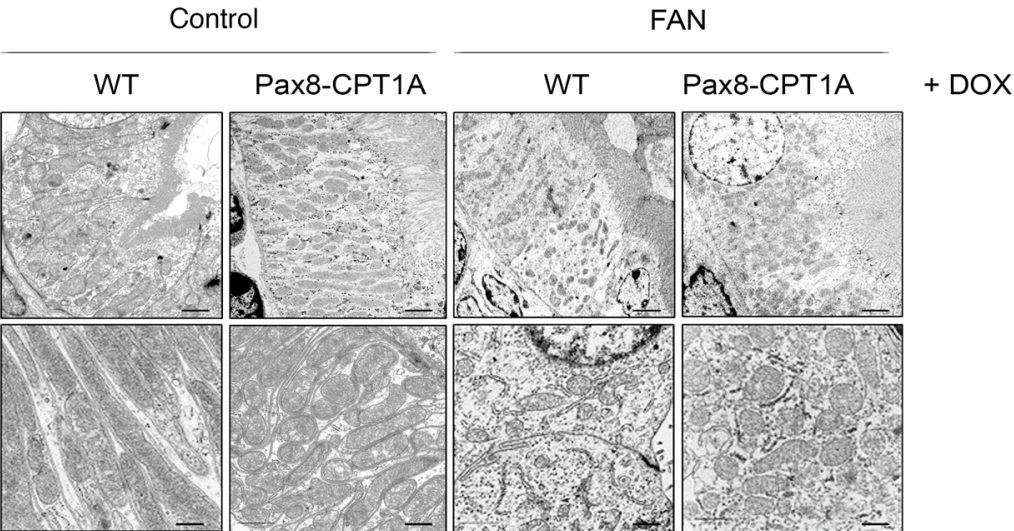

B

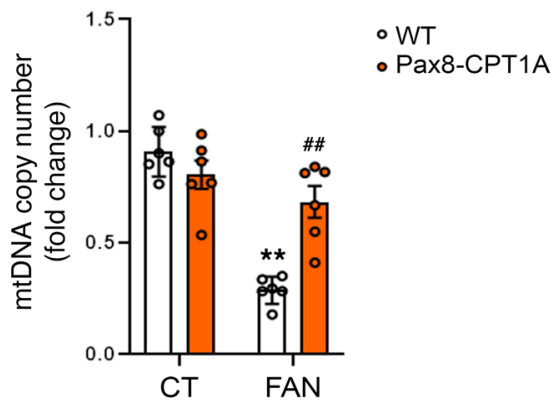

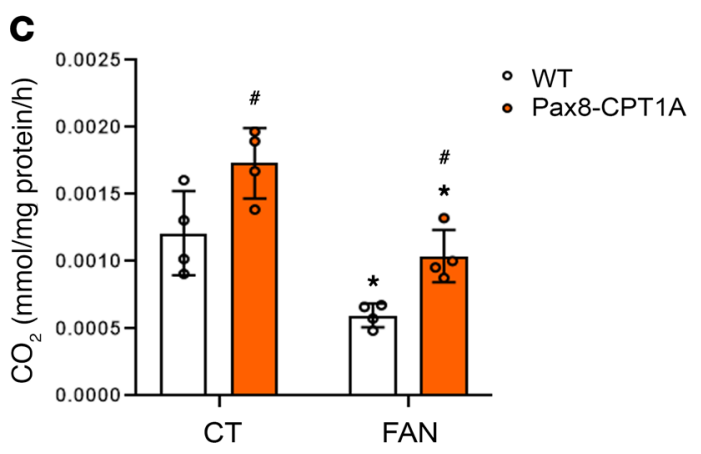

E

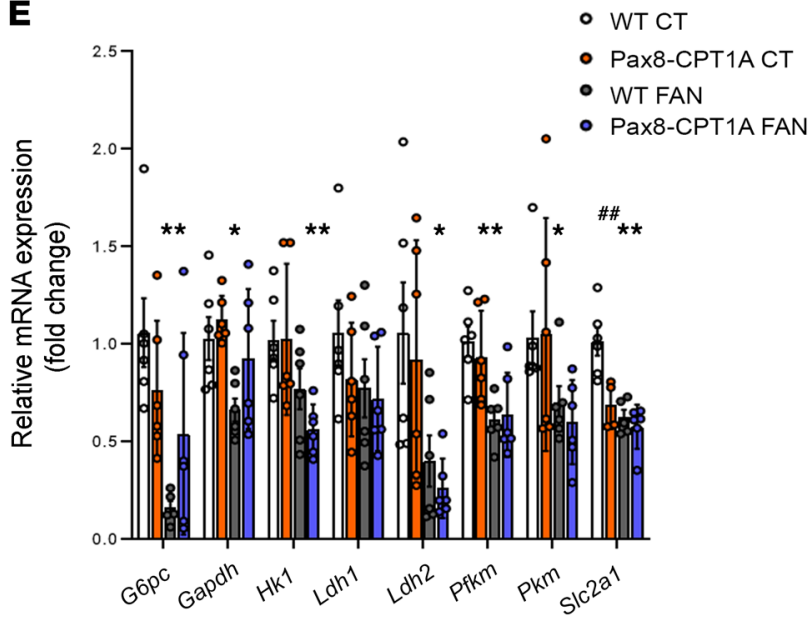

D

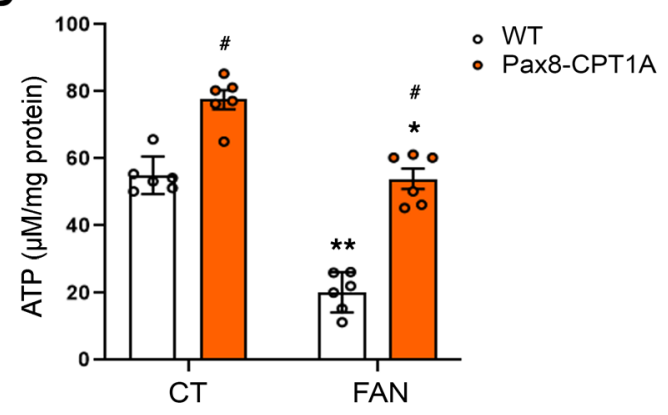

$\mathbf{F}$

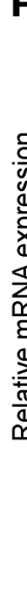

- WT CT

- Pax8-CPT1A CT

- WT FAN

- Pax8-CPT1A FAN

Figure 3. CPT1A prevents impaired mitochondrial morphology and FAO defect in FAN-induced kidney fibrosis. (A) Representative electron microscopy images of cortical proximal tubules from control and Pax8-CPT1A mice subjected to FAN after doxycycline induction. Scale bars: $10 \mu \mathrm{m}$ (upper panels), $100 \mathrm{~nm}$ (lower panels). (B) Mitochondrial DNA copy number (mtDNA) was determined in kidneys of WT and Pax8-CPT1A mice in the FAN model. Bar graphs represent the mean \pm SEM of fold changes $\left(n=6\right.$ mice). ${ }^{* *} P<0.01$ compared with their corresponding control (CT) kidneys; ${ }^{\# \#} P<0.05$ compared with kidneys from WT mice with the same experimental condition. (C) Radiolabeled palmitate-derived $\mathrm{CO}_{2}$ was determined after incubation of ${ }^{14} \mathrm{C}$-palmitate with kidney tissue from WT and Pax8-CPT1A mice in the FAN model after doxycycline induction. (D) ATP levels in total kidney tissue determined in mice subjected to FAN model. (C and D) Bar graphs represent the mean \pm SEM $\left(n=4\right.$ mice). ${ }^{*} P<0.05$, ${ }^{* *} P<0.01$ compared with their corresponding CT kidneys; ${ }^{\#} P<0.05$ compared with kidneys from WT mice with the same experimental condition. (E and $\mathbf{F}$ ) mRNA levels of glucose utilization-associated genes (E) and peroxisomal/mitochondrial function-associated genes (F) were determined by qRT-PCR using TaqMan qPCR probes in kidneys from CT and FA-treated (FAN) WT and Pax8-CPT1A mice after doxycycline induction. (E and F) Bar graphs represent the mean \pm SEM of fold changes $\left(n=6\right.$ mice). ${ }^{*} P<0.05,{ }^{* *} P$ $<0.01$ compared with their corresponding CT kidneys; ${ }^{\#}<0.05$, ${ }^{\#} P<0.01$ compared with kidneys from WT mice with the same experimental condition. Statistical significance between 2 independent groups was determined using nonparametric 2-tailed Kruskal-Wallis test. For detailed gene nomenclature, see Supplemental Table 4. 
served sensor of intracellular metabolic status and plays a critical role in systemic energy homeostasis (8). To determine whether FAO-GOF in the kidneys of CPT1A-KI mice was associated with changes in AMPK activation as a consequence of increased ATP levels, phosphorylation of AMPK was analyzed by immunoblot. In the FAN model, obstructed kidneys presented increased AMPK phosphorylation protein levels compared with contralateral kidneys. Importantly, increasing CPT1A levels attenuated AMPK phosphorylation in fibrotic kidneys in the FAN model (Supplemental Figure 8, A and B). The levels of phosphorylated acetyl-CoA carboxylase, directly dependent on AMPK, changed accordingly. Similar data were obtained in the UUO and ADN models (Supplemental Figure 8, C-F). Overall, we found that increased FAO associated with overexpression of CPT1A improved mitochondrial respiration in the context of reduced glycolysis. Moreover, it is likely that the enhancement of ATP production related to FAO reins in AMPK activation triggered by chronic kidney damage, independent of the model employed.

Overexpression of CPT1A modifies the cellular inflammatory profile and abrogates TGF- $\beta$-associated epithelial cell damage. Studies in patients and in animal models show a strong correlation between infiltrated macrophage polarization and the extent of fibrosis (9). In the FAN model, we found that overexpression of CPT1A correlated with a decreasing trend in macrophage influx (Figure 5, A and B), and flow cytometry analysis revealed a reduced proportion of the renal proinflammatory M1 subpopulation (Figure 5, C and D). In the UUO (Supplemental Figure 9, A and B) and ADN (Supplemental Figure 5, E and F) models, the CPT1Ainduced decrement in macrophage influx was also confirmed. By contrast, the macrophage subpopulation positive for CD86 (M1) was higher than that for CD206 (M2) in fibrotic kidneys 3 and 7 days after UUO, whereas CPT1A overexpression did not affect this shift (Supplemental Figure 9, C-F). However, the M2 macrophage subpopulation was increased in fibrotic kidneys from CPT1A-KI mice compared with the WT ones after 7 days of UUO (Supplemental Figure 9, E and F). Infiltration of the macrophage subpopulation positive for both CD86 and CD206 (M1/M2) observed in obstructed kidneys from WT mice 3 or 7 days after UUO was also enhanced by CPT1A overexpression (Supplemental Figure 9, D and $\mathrm{F}$ ). In addition, the abundance of this M1 subpopulation was lower in FAN-induced fibrotic kidneys than in UUO-associated ones (Figure 5D and Supplemental Figure 9, D and F). These data suggest that the changes in the degree of macrophage infiltration and relative contributions of macrophage subpopulations are dependent on the model of kidney injury.

Inflammation is a major hallmark of renal fibrosis, especially in the early stages (9). Consistently, kidney infiltration by CD3+ T lymphocytes after FAN was reduced in CPT1A-KI mice compared with WT mice (Supplemental Figure 2, D and E). Thus, we analyzed the profile of prototypical molecules related to inflammation in the kidneys of mice overexpressing CPT1A in the context of renal damage. In the FAN model, we found a lower expression of inflammation-related markers, including the cytokines $I L-1 b$, $I L-6$, and Tnf- $\alpha$ (Figure 5E), in contrast to the findings in the 7-day UUO model (Supplemental Figure 10C). Consistently, cells from kidneys of mice overexpressing CPT1A presented a reduced population of damaged epithelial cells (CD45- EpCAM+ CD24+; ref.
10) compared with those of WT mice in both the FAN and 7-day UUO models (Figure 6, A and B and Supplemental Figure 10, A and B). In the FAN model, the levels of 3 necroptosis markers, receptor-interacting serine/threonine-protein kinase (RIPK1), mixed lineage kinase domain-like pseudokinase (MLKL), and phosphorylated RIPK-3, were significantly blunted in the presence of CPT1A overexpression (Figure 6, C-E). Moreover, there was also a reduced expression of the apoptotic markers Apaf1, Bax, and $\mathrm{Bcl} 2$ (Figure 6F). A similar pattern regarding the expression of apoptotic markers was observed in the 7-day UUO model (Supplemental Figure 10D).

Epithelial cell dedifferentiation is a process associated with the transformation of a terminal cellular phenotype into one with relatively higher potential of differentiation into more than one cell type (11). This dedifferentiation is characterized by the loss of epithelial markers and acquisition of mesenchymal features, a process known as epithelial-mesenchymal transition (EMT, ref. 12), the role of which in kidney fibrosis remains controversial. We studied the expression of E-cadherin, whose loss is considered a representative feature of EMT, in the setting of CPT1A overexpression and found no significant differences in renal tubular expression in any of the 3 models employed (Supplemental Figure 11A). A similar pattern was observed when further EMTassociated markers were analyzed in the FAN model. Only the EMT-associated increased expression of Snail was prevented in the kidneys of CPT1A-KI mice (Supplemental Figure 11B). We also tested whether enhancing renal epithelial FAO had a protective role in TGF- $\beta$-induced transformation of epithelial cells into a cellular fibrotic phenotype. Primary kidney epithelial cells isolated from CPT1A-KI mice and treated with TGF- $\beta 1$ for 48 hours showed a marked reduction in the increase of these EMTassociated markers compared with the cells isolated from WT mice (Supplemental Figure 11C). Consistently, HKC-8 cells transduced with CPT1A exhibited a lower expression of collagen (Supplemental Figure 11D) and fibronectin (Supplemental Figure 11E). This set of data indicate that the protective action of CPT1A in kidney fibrosis operates, at least in part, through the attenuation of TGF- $\beta 1$-induced TEC transformation.

Overexpression of CPT1A after damage markedly attenuates the fibrotic phenotype. To investigate whether restoration of FAO-GOF after injury could also protect from kidney damage, we started doxycycline administration after fatty acid injection and confirmed that CPT1A levels increased gradually in the CPT1A-KI mice (Supplemental Figure 12, A-C). We observed that the fibrotic area and renal function impairment were significantly reduced in the CPT1A-KI model (Figure 7, A-C). The expression of the profibrotic markers Col1a1 and $F n 1$ was also significantly reduced in the CPT1A-KI mice (Figure 7D). TECs from CPT1A-KI mice also exhibited a more favorable mitochondrial bioenergetic profile after damage, which was reflected by a higher ATP production rate and basal and maximum OCRs (Figure 7, E and F). This data reinforces the potential of FAOGOF to combat kidney fibrosis even when renal damage has already been initiated.

An increase in plasma acylcarnitines and a reduction of CPT1A levels is present in patients with CKD. To explore the importance of FAO in a clinical context, we analyzed a cohort of 686 patients 
A
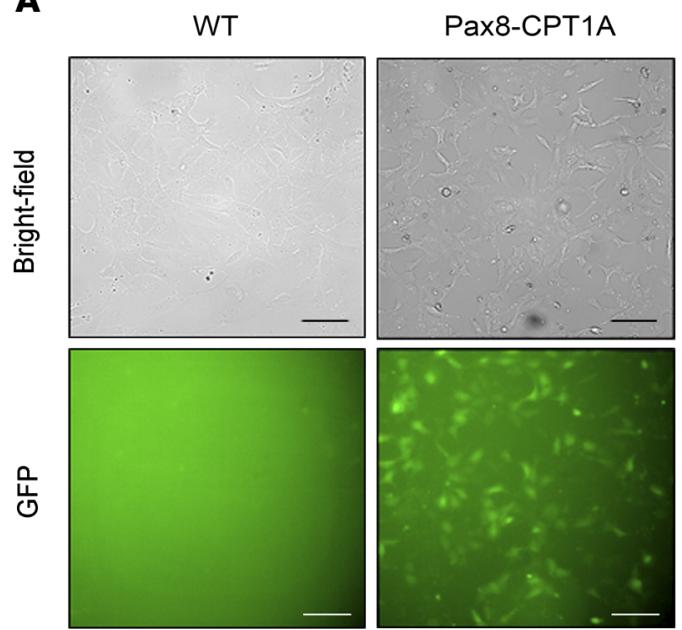

C

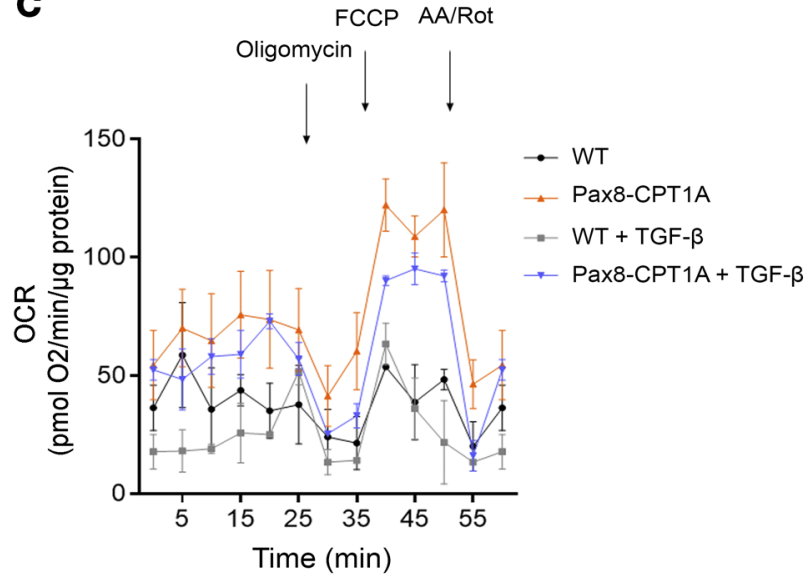

D

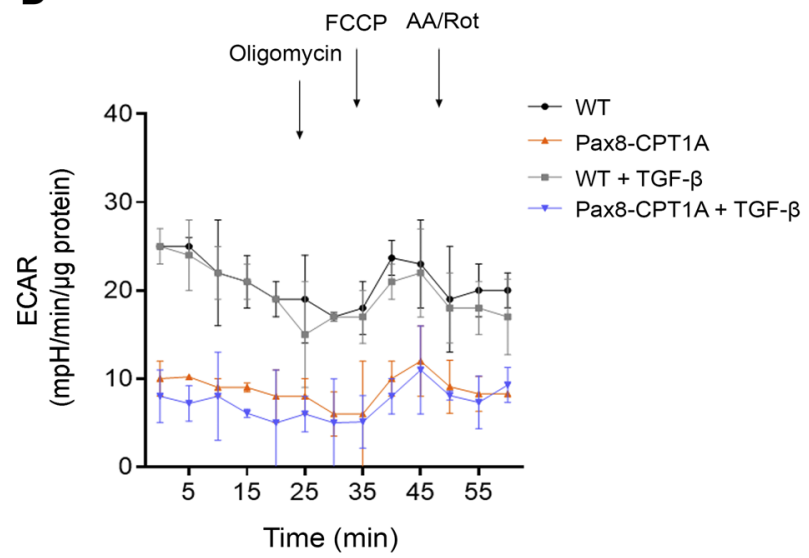

B
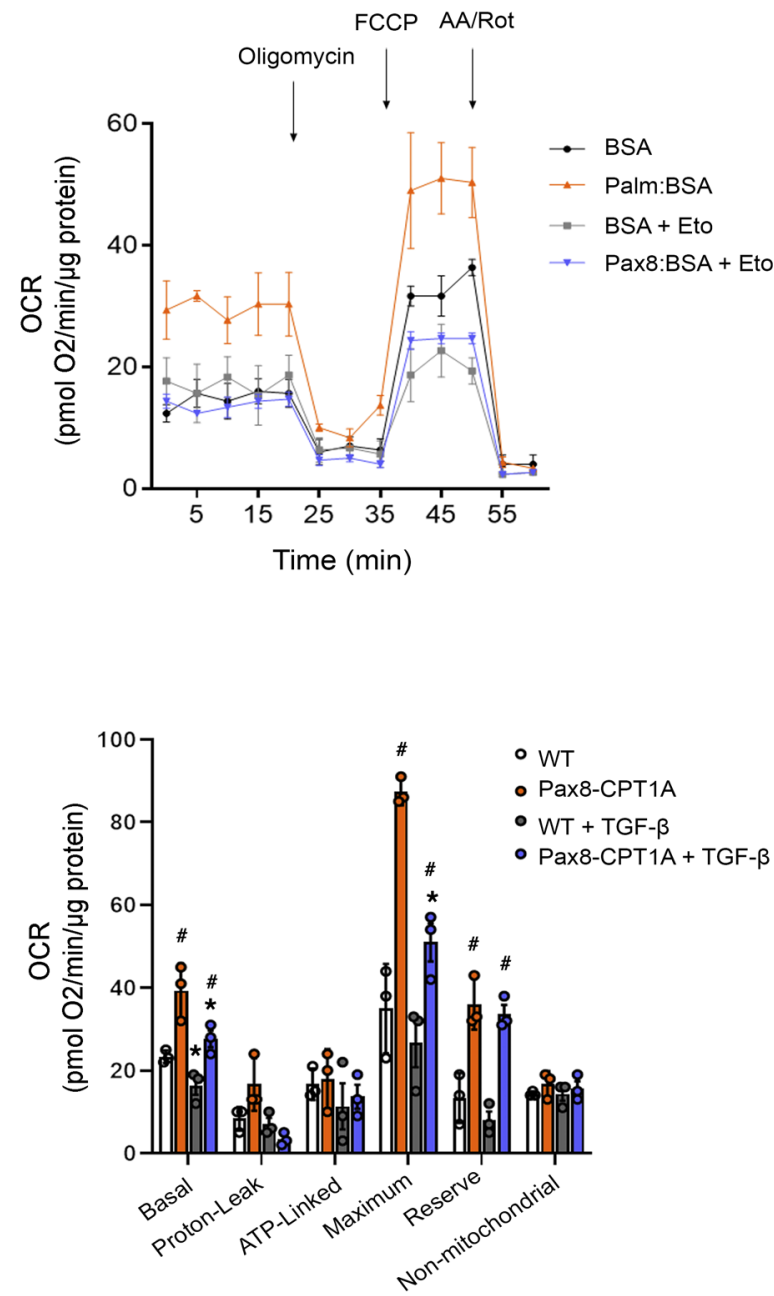

E

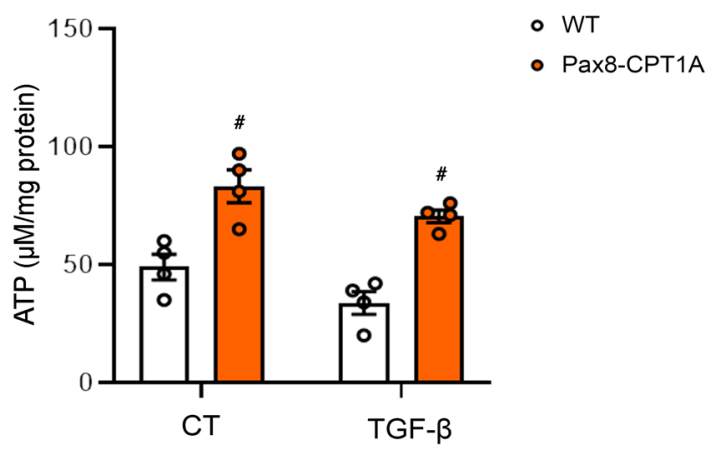

Figure 4. CPT1A overexpression prevents TGF- $\beta 1$-induced FAO impairment. (A) Bright field or GFP immunofluorescence images of primary kidney epithelial cells (TECs) isolated from kidneys of WT and Pax8-CPT1A mice. Scale bar: $20 \mu \mathrm{m}$. (B) Oxygen consumption rate (OCR) of TECs from WT mice was measured with a Seahorse XF24 Extracellular Flux Analyzer. Where indicated, cells were pretreated with palmitate-BSA FAO substrate (200 $\mu$ M) or the CPT1 inhibitor etomoxir (Eto, $400 \mu \mathrm{M})$. Oligomycin $(1 \mu \mathrm{M})$, FCCP $(3 \mu \mathrm{M})$, and a combination of antimycin $\mathrm{A}(1 \mu \mathrm{M})$ and rotenone $(1 \mu \mathrm{M})$ (AA/Rot) were injected sequentially at the time points indicated. Each data point represents the mean \pm SEM of 4 independent experiments, each performed in triplicate. (C) OCR of TECs from WT and Pax8-CPT1A mice was measured with a Seahorse XF24 Extracellular Flux Analyzer. Bar graphs (right panel) show the rates of OCR associated with basal, proton-leak, ATP-linked, maximum, reserve capacity, and nonmitochondrial respiratory statuses. ${ }^{*} P<0.05$ compared with their corresponding control (CT) TECs; ${ }^{\# P}<0.05$ compared with TECs from WT mice with the same experimental condition. (D) Extracellular acidification rate (ECAR) of cells treated as in A. Each data point represents the mean \pm SEM of 4 independent experiments, each performed in triplicate. (E) ATP levels of TECs from WT and Pax8-CPT1A mice. Bar graphs represent the mean \pm SEM ( $n=4$ mice per group). ${ }^{\#} P<0.05$ compared with kidneys from WT mice with the same experimental condition. Statistical significance between 2 independent groups was determined using nonparametric 2 -tailed Kruskal-Wallis test. 
A

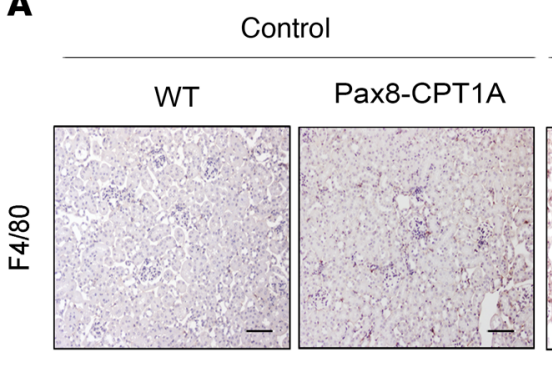

FAN

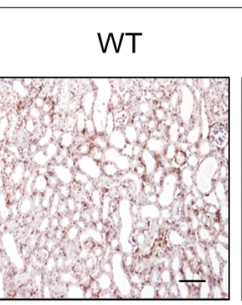

Pax8-CPT1A
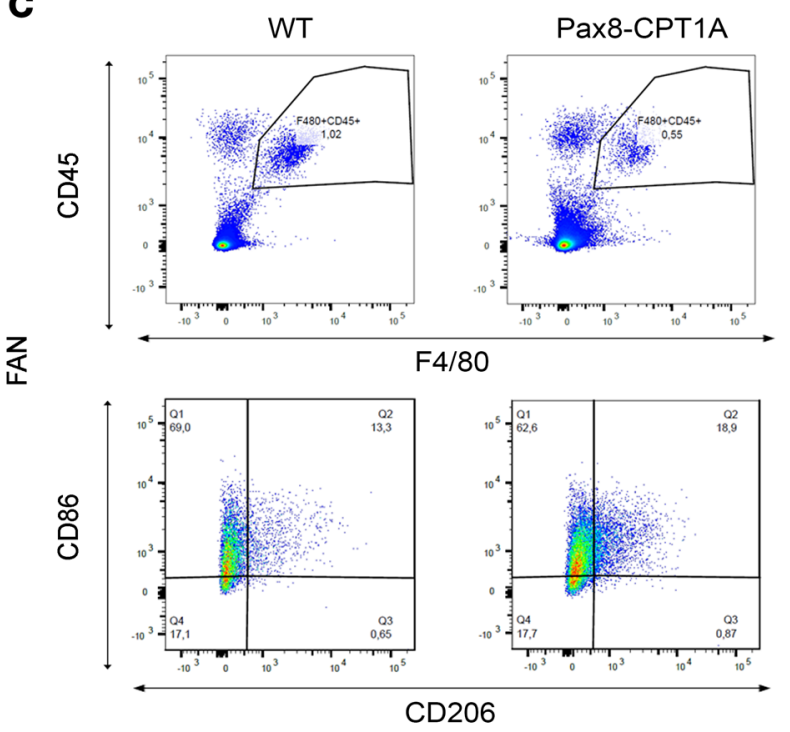

Pax8-CPT1A + DOX

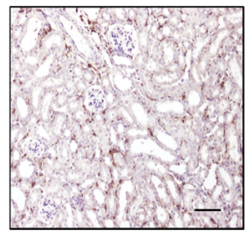

+ DOX
B

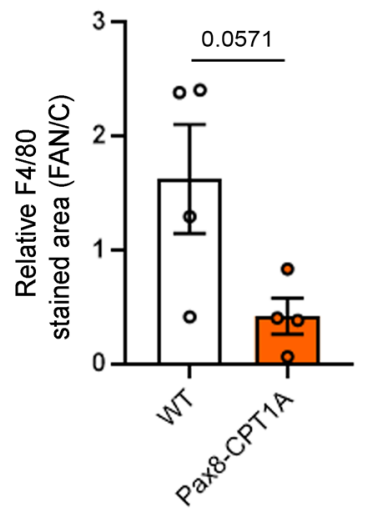

D

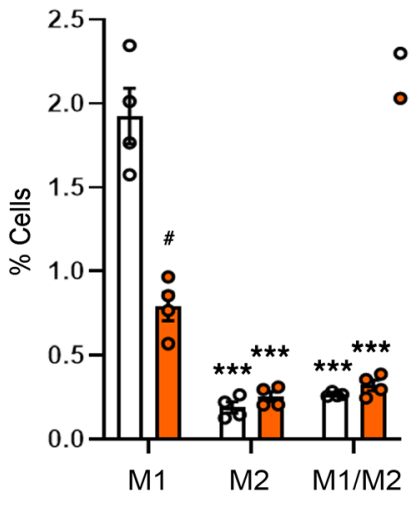

- WT FAN

- Pax8-CPT1A FAN

E

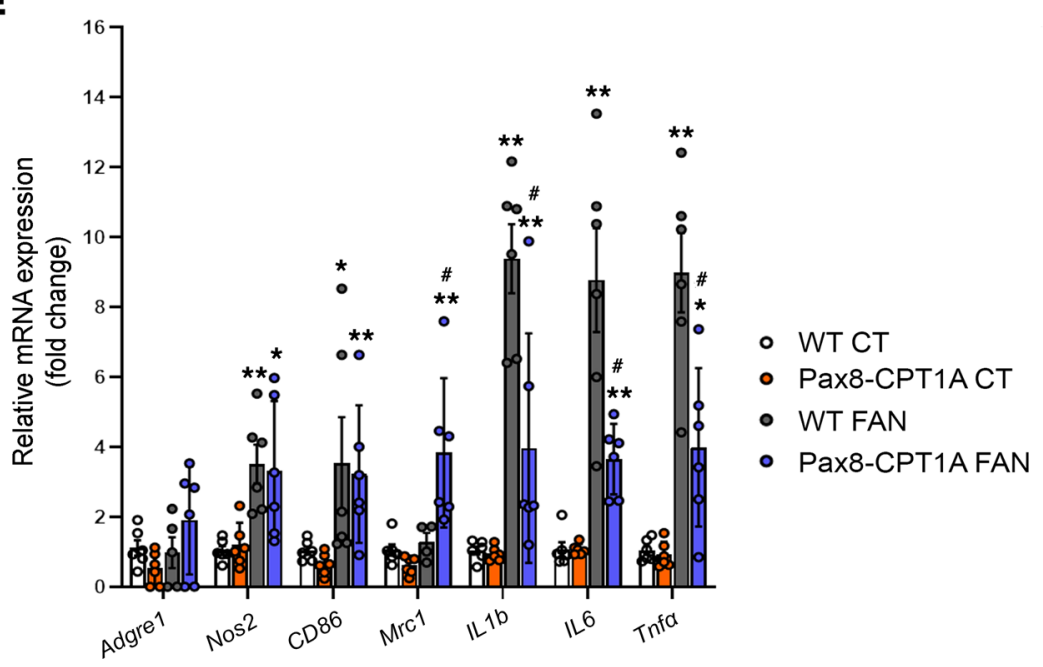

Figure 5. Overexpression of CPT1A reduces M1 macrophage infiltration in the FAN model. (A) Representative micrographs of 1 mouse per group showing the expression of F4/80 in kidney sections of mice treated as described above. Scale bar: $50 \mu \mathrm{m}$. (B) Bar graph represents the quantification of the percentage of $\mathrm{F} 4 / 80$ + stained area in FA-treated mouse kidneys (FAN). Bar graphs represent the mean $\pm \mathrm{SEM}, n=4$ mice. (C) Representative multiparameter flow cytometry dot plots showing the expression of CD45 and F4/80 in kidney cells from WT and Pax8-CPT1A mice subjected to FAN after doxycycline induction (upper panels) (1 mouse per group is represented). CD86 and CD206 were used to determine the proportion of M1 and M2 macrophage subpopulations, respectively, in the total macrophage population ( $\mathrm{F} 4 / 80+, \mathrm{CD} 45+)$ (lower panels). Numbers in quadrants indicate cell proportions in percentage of cells that express both markers. (D) Bar graph represents the percentage of kidney cells expressing CD86 (M1), CD206 (M2), or both (M1/M2) markers. Data represent mean \pm SEM $\left(n=4\right.$ mice). ${ }^{* * *} P<0.001$ compared with M1 subpopulation in damaged kidneys from WT mice; ${ }^{*} P<0.05$ compared with corresponding cell subpopulation in damaged kidneys from WT mice. (E) mRNA levels of inflammation-associated genes were determined by qRT-PCR using TaqMan qPCR probes in kidneys from control (CT) and FA-treated (FAN) WT and Pax8-CPT1A mice after doxycycline induction. Bar graphs represent the mean \pm SEM of fold changes $\left(n=6\right.$ mice). ${ }^{*} P<0.05,{ }^{*} P<0.01$ compared with their corresponding CT kidneys; $P<0.05$ compared with kidneys from WT mice with the same experimental condition. Statistical significance between 2 independent groups was determined using nonparametric 2 -tailed Mann-Whitney $U$ test; more than 2 groups were compared with Kruskal-Wallis test. For detailed gene nomenclature, see Supplemental Table 4. 
A
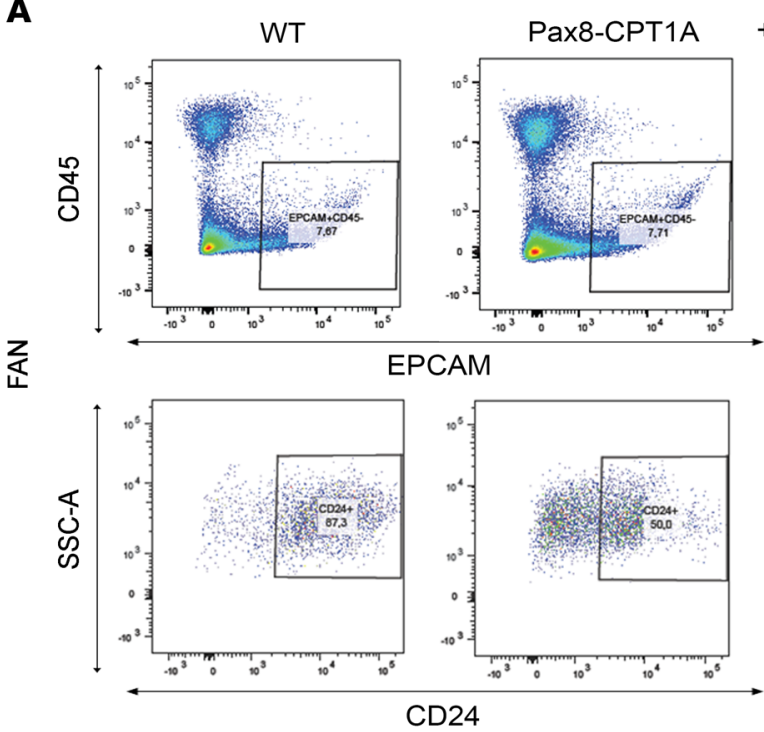

C

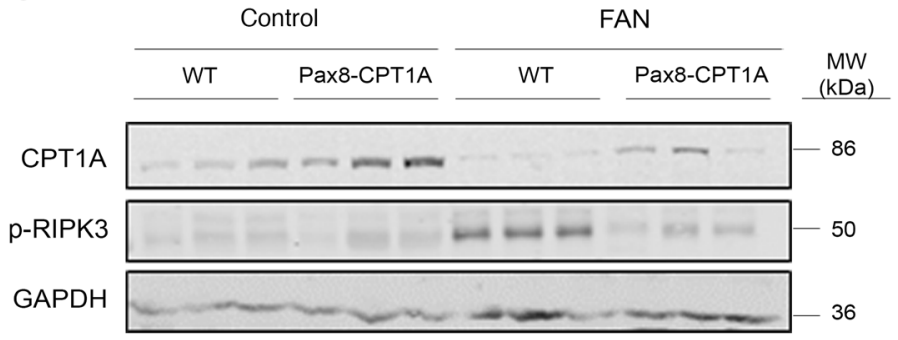

$\mathbf{E}$

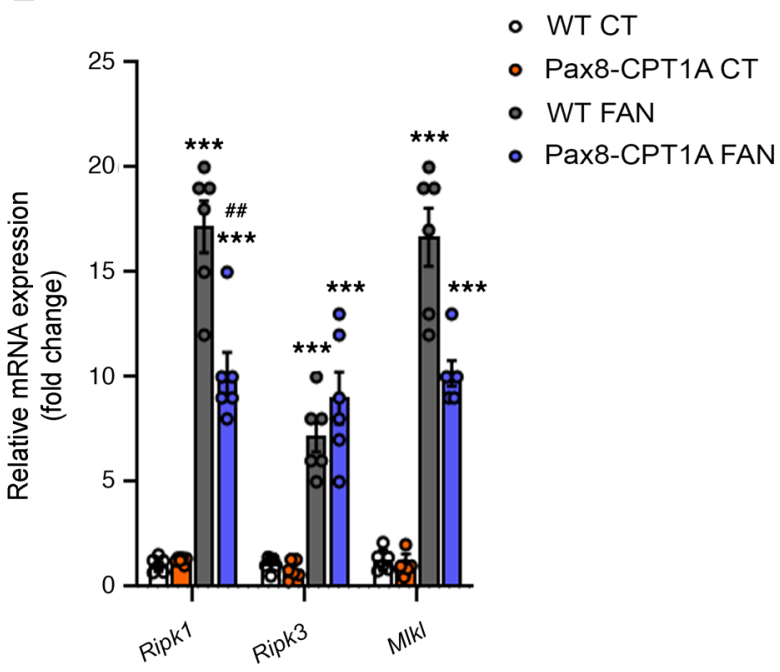

B

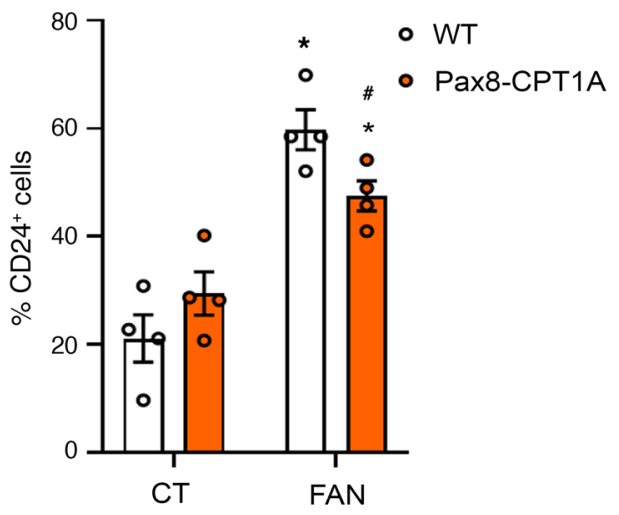

D

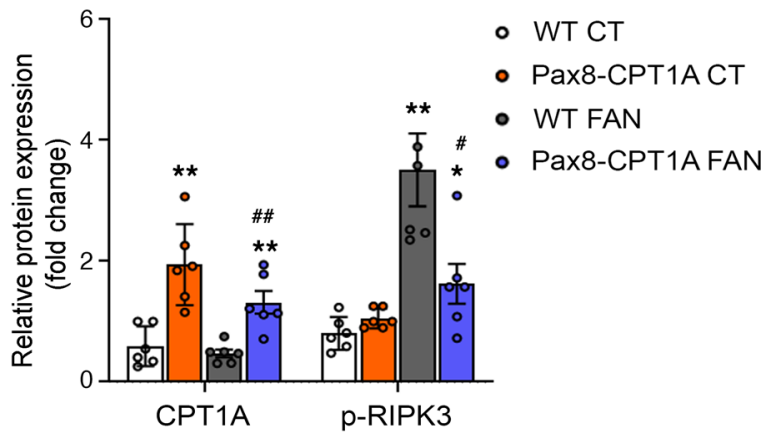

$\mathbf{F}$

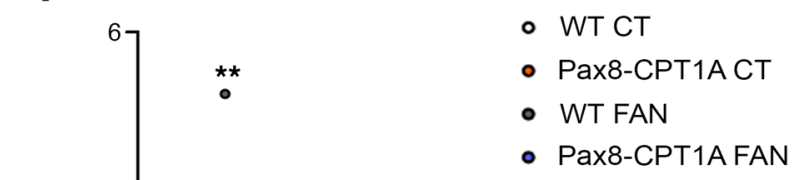


Figure 6. CPT1A overexpression reduces epithelial cell damage in the FAN model. (A) Representative flow cytometry dot plots from obstructed kidneys of WT and Pax8-CPT1A mice subjected to FAN after doxycycline treatment. Cells were gated for CD45 negative/epithelial cell adhesion molecule (EpCAM) positive (upper panels) and selected for the presence of CD24 (lower panels). Numbers in quadrants indicate cell proportions. (B) Bar graphs show the percentage of kidney cells positive for CD24. Data represent the mean \pm SEM ( $n=4$ mice). ${ }^{*} P<0.05$ compared with their corresponding control (CT) kidneys; ${ }^{P} P<0.05$ compared with damaged kidneys in WT mice. (C and $\mathbf{D})$ Representative immunoblots and densitometries corresponding to CPT1A and phosphorylated RIPK3 protein levels in kidneys as in $\mathbf{A}$ ( $n=3$ mice). ${ }^{*} P<0.05,{ }^{*} P<0.01$ compared with their corresponding CT kidneys; ${ }^{\#} P<0.05,{ }^{\# \#} P<0.01$ compared with kidneys from WT mice with the same experimental condition. (E) mRNA levels of RIPK1, RIPK3, and MLKL were determined by qRT-PCR in kidneys as in $\mathbf{A}$. Bar graphs represent the mean \pm SEM of fold changes ( $n=6$ mice). ${ }^{* *} P<$ 0.001 compared with their corresponding CT kidneys; ${ }^{\#} P<0.01$ compared with kidneys from WT mice with the same experimental condition. (F) mRNA levels of apoptosis-associated genes were determined by qRT-PCR using TaqMan qPCR probes in kidneys from CT and FA-treated (FAN) WT and Pax8-CPT1A mice after doxycycline induction. Bar graphs represent the mean \pm SEM of fold changes $\left(n=6\right.$ mice). ${ }^{*} P<0.05$, ${ }^{*} P<0.01$ compared with their CT kidneys; $\# \#<0.05$ compared with kidneys from WT mice with the same experimental condition. Statistical significance between 2 independent groups was determined using nonparametric 2-tailed Kruskal-Wallis test. For detailed gene nomenclature, see Supplemental Table 4.

with CKD and diabetes pertaining to the PREDIMED study (ref. 13, see Supplemental Table 7 for details). In patients whose renal function parameters were available $(n=686)$, we determined the levels of acylcarnitines. We found inverse correlation between glomerular filtration rate (GFR) and short-chain (c2-c7) and medium-chain (c8-c14) acylcarnitine levels (Figure $8, \mathrm{~A}$ and B and Supplemental Tables 8 and 9) in patients with a GFR under $60 \mathrm{~mL} / \mathrm{min}$. Thus, CKD patients with a higher GFR showed less accumulation of short- and medium-chain acylcarnitines (Figure 8, D, E, and G), with no correlation in the case of long-chain (c16-c26) acylcarnitines (Figure 8, C and F). In a different cohort of CKD patients (see Supplemental Table 10 for details), we found a positive correlation between tubule CPT1A expression levels and eGFR (Figure 8, $\mathrm{H}$ and I) in an RNA-Seq study from different pathological backgrounds. The degree of fibrosis also correlated significantly with declining CPT1A levels (Figure 8J). The increase in the levels of acylcarnitines and the reduction in the levels of the limiting-step enzyme responsible for their metabolism (CPT1A) most likely reflect a decreased FAO capacity associated with CKD. Thus, these results underscore the relevance of reduced FAO in CKD and support the importance of its GOF to combat kidney fibrosis, as demonstrated in our experimental model.

\section{Discussion}

Kidney fibrosis is critically linked to metabolic failure in TECs (14). We demonstrated that genetic FAO-GOF was sufficient to provide significant mitigation of fibrosis development in 3 experimental models of chronic kidney damage. Moreover, FAO-GOF was also therapeutically effective when reinstated after kidney damage. Although FAO is a complex process depending on several biochemical steps and enzymatic systems, CPT1A is ratelimiting because of its key role for shuttling medium and long acyl
CoA chains into the mitochondrial matrix with the concourse of L-carnitine (15). To date, no drugs are available to specifically activate CPT1 and hence, pharmacological studies are not devoid of limitations. Homozygous CPT1A deficiency is lethal in the mouse (16); tissue-specific CPT1A-KO models for the endothelium (17), pancreatic $\alpha$ cells (18), and intestinal stem cells (19) have been generated. None of these studies addressed potential consequences on organ fibrosis. The FAO-GOF genetic model herein described is likely one of the first direct demonstrations of the potential of this strategy to treat kidney fibrosis. The study was performed in heterozygous mice in an attempt to avoid nonphysiological scenarios due to CPT1A overdosing (20). Mitigation of renal fibrogenesis was reflected in a reduction of fibrotic markers, histological improvement, and amelioration of renal function. Slight differences in the magnitude of changes are most likely due to variation in the intensity of the inflammatory or fibrotic components among models. Although no experimental model recapitulated with close fidelity human CKD, the significant correlation between advanced kidney disease and 2 signatures of FAO reduction (increased acylcarnitines and low CPT1A) in 2 large patient cohorts attests to the clinical relevance of an FAO-related major metabolic disturbance in human CKD (21). Adjustment for age, sex, diabetic state, and albumin/creatinine ratio indicates that renal function was likely the variable accounting for these differences, even though the contribution of other tissues, such as adipose, cannot be completely excluded. The fact that we could not evaluate levels of CPT1A and acylcarnitines simultaneously in any of the 2 cohorts poses some limitations for a clear-cut interpretation. We found correlation between GFR and short- and medium- but not long-chain acylcarnitine levels, as could be initially expected. In a detailed study, Afshinnia et al. found higher plasma abundance of long-chain free fatty acids coupled with a significantly lower long-to-intermediate acylcarnitine ratio (a marker of impaired $\beta$-oxidation) in severe stages of CKD, but CPT1A levels were not determined (21). Increased levels of short and middle acylcarnitines in the diabetic cohort with CKD may be explained by alterations in the expression or activity of FAO-related enzymes, including CPT1A (3), as accumulation of short- and middle-chain acylcarnitines may result from incomplete FAO of long-chain acylcarnitines (22). Further, the systemic clearance of acylcarnitines may be differentially impaired in this population, also affecting their biodistribution $(23,24)$.

The high energetic demand of renal TECs, required for their performance of multiple functions related to the homeostasis of the internal milieu, dictates their dependence on intact mitochondrial function. We showed that FAO-GOF, attained by overexpressing tubular CPT1A (in both animals and cells), restored mitochondrial mass and architecture, enhanced OCR, and increased ATP production in conditions of renal damage. This appeared to occur at the expense of glycolysis because ECAR was significantly lower under conditions of CPT1A overexpression. The reciprocal regulation between FAO and glycolysis, the Randle cycle (25), has been demonstrated in the muscle and heart; our data showed that it also takes place in renal tubular cells. Alternatively, it is possible that TECs are consuming lactate as the main fuel for gluconeogenesis, a process in which the kidney plays an essential role (26). The fundamental question as to whether metabolism dictates 
A

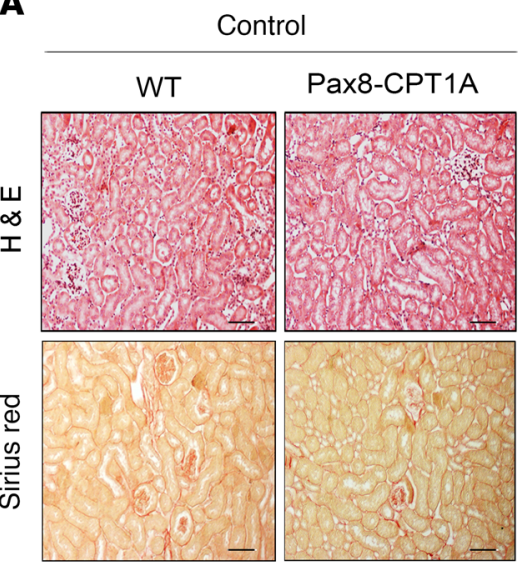

$\frac{\text { FAN }}{\text { WT } \quad \text { Pax8-CPT1A }}+$ DOX

B
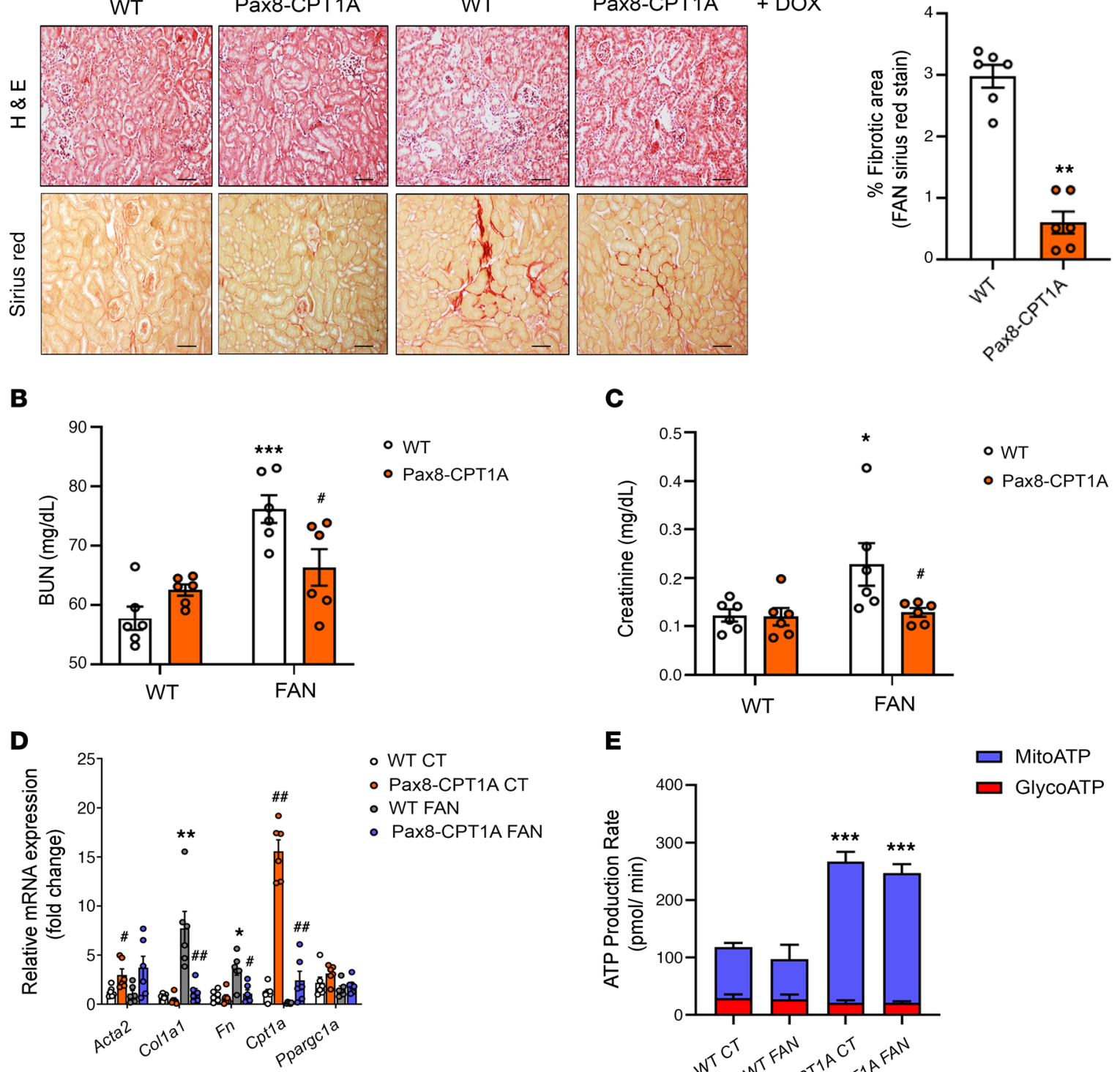

- WT CT

- Pax8-CPT1A CT

- WT FAN

- Pax8-CPT1A FAN

C

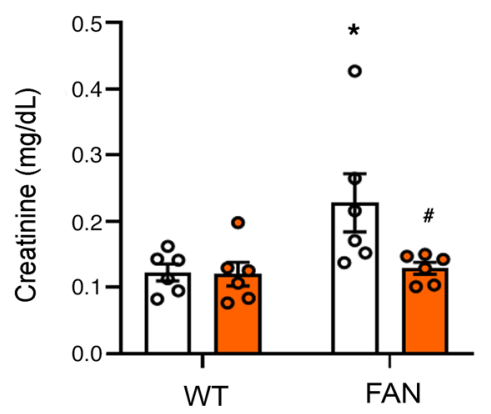

E

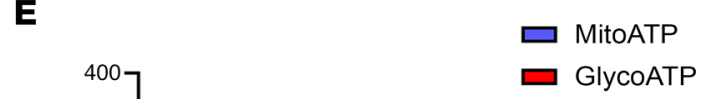

$\mathbf{F}$
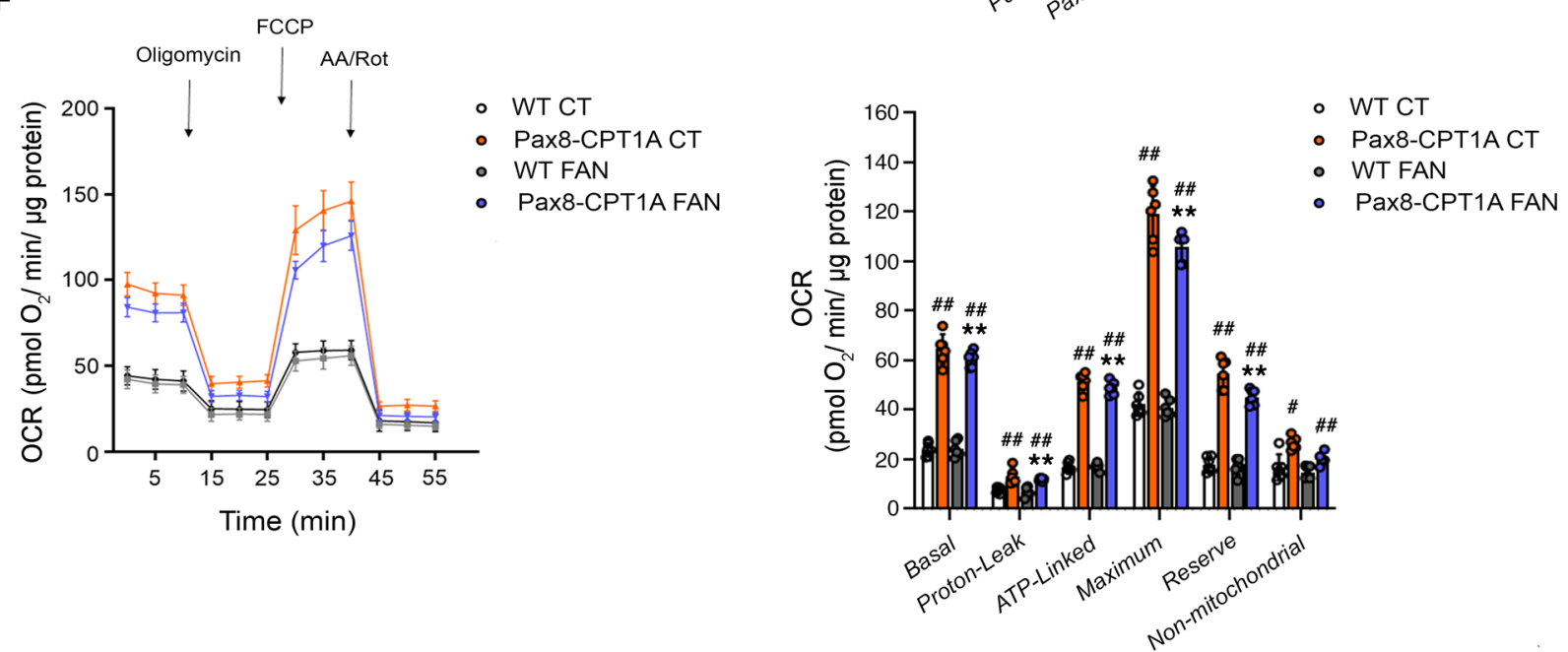
Figure 7. CPT1A upregulation after FA-induced renal disease mitigates FAN-associated kidney function deterioration, renal fibrosis, and FAO defects. (A) Representative microphotographs of H\&E (upper panels) and Sirius red (lower panels) staining of kidneys from WT and Pax8-CPT1A mice subjected to FAN prior to doxycycline (Dox) (Supplemental figure 12A). Scale bars: $50 \mu \mathrm{m}$. Quantification of Sirius red staining represents the mean $\pm \mathrm{SEM}, n=6$ mice. ${ }^{* *} P<0.05$ compared with FAN kidneys in WT mice. (B and C) Serum BUN (B) and creatinine (C) levels of WT and Pax8-CPT1A mice subjected to FAN as in A. Data represent the mean \pm $\operatorname{SEM}\left(n=6\right.$ mice). ${ }^{*} P<0.05,{ }^{* * *} P<0.001$ compared with respective control (CT) condition; ${ }^{\# P}<0.05$ compared with WT mice with the same condition. (D) mRNA levels of $\alpha$-SMA, Col1 $\alpha 1$, FN, CPT1A, and Ppargc1a determined by qRT-PCR in kidneys of WT and Pax8-CPT1A mice subjected to FAN as in $\mathbf{A}$. Bar graphs represent the mean \pm SEM of fold changes ( $n=6$ mice). ${ }^{*} P<0.05,{ }^{* *} P<0.01$ compared with corresponding CT kidneys; ${ }^{\#} P<0.05,{ }^{\#} P<0.01$ compared with kidneys from WT mice with the same condition. ( $E$ and $\mathbf{F}$ ) ATP production rate of TECs from WT and Pax8-CPT1A mice subjected to FAN as in $\mathbf{A} .{ }^{* *} P<0.001$ compared with TECs from WT mice with the same condition. (F) Oxygen consumption rate (OCR) of TECs from WT and Pax8-CPT1A mice subjected to FAN as in A. Bar graphs (right panel) show the rates of OCR as in Figure 4 C. ${ }^{* *} P<$ 0.01 compared with their corresponding control (CT) TECs; ${ }^{P} P<0.05$ and $\# \#<0.01$ compared with TECs from WT mice with the same condition. Statistical significance between 2 independent groups was determined using nonparametric 2-tailed Mann-Whitney $U$ test; more than 2 groups were compared with Kruskal-Wallis test.

phenotype is in part answered here, as our data indicate that an increased capacity of the TEC to meet its energy challenges is able to both prevent and revert kidney fibrosis. Thus, efforts directed at enhancing FAO in the early stages of fibrosis should very likely pay off to combat human CKD.

\section{Methods}

Generation of a transgenic mouse model for the inducible Cpt1a gene. Generation of an inducible conditional transgenic mouse model for Cpt1a overexpression in the renal epithelium was based on a second generation Tet-On system with site-specific recombination in embryonic stem (ES) cells $(27,28)$. Mice harboring the transgene of the Cpt1a gene under the control of the tetracycline-responsive promoter element (tetO-Cpt1a mice) were generated in the transgenic mice core unit from the National Cancer Research Center (CNIO; Madrid, Spain). Mice were generated by diploid blastocyst injection. Blastocysts were harvested at 3.5 days after coitum from C57/BL6-J strain females. Between 10 and $15 \mathrm{KH} 2 \mathrm{ES}$ cells were injected per blastocyst. Approximately 30 injected blastocysts were transferred into pseudopregnant CD1 strain-recipient females (transgenic mice core unit, CNIO). Chimeras from the litter with a high percentage of agouti coat color (>80\%) were then crossed with C57/BL6-J mice to evaluate germline transmission. Next, these mice were crossed with mice providing renal epithelial tissue specificity, Pax8-rtTA (The Jackson Laboratory). Male tetO-Cpt $1 \mathrm{a}^{\text {tg } / 0}$ mice were bred with Pax8-rtTA ${ }^{\text {tg } / 0}$ female mice to generate double heterozygous Pax8-rtTA ${ }^{\mathrm{tg} / 0}$ tetO-Cpt1 $\mathrm{a}^{\mathrm{tg} / 0}$ mice and their control littermates in a B6N 129Sv mixed genetic background. Extrarenal expression only occurs at the level of the thyroid, and this fact does not interfere with any kidney phenotype (5).

Molecular cloning and gene targeting in ES cells. The CPT1A transgene containing the 5'UTR of the Cpt1a gene, the Cpt1a open reading frame (ORF) (GenBank accession number NM_013495.2), the 2A self-cleaving peptide (P2A), the gene encoding for GFP, and the 3'UTR of the Cpt1a gene were cloned into a unique EcoRI site of pBS31 vector (OriGene Technologies). This vector contains the phosphoglycerate kinase (PGK) promoter followed by an ATG start codon and an FRT recombination site, a splice acceptor-double polyA cassette, the tetracycline operator with a CMV minimal promoter, and an SV40 polyA signal, assembled in Bluescript. By using FRT/flippase-mediated recombination system $(27,28)$, the CPT1A transgene was targeted into the downstream region of the collagen 1a1 (Col1a1) locus of KH2 ES cells containing an frt-flanked PGK-neomycin-resistance gene followed by a promoterless, ATG-less hygromycin-resistance gene. These cells also contained the M2-rtTA under control of the endogenous ROSA26 promoter (29). For gene targeting, $50 \mu \mathrm{g}$ pBS31 vector and $25 \mu \mathrm{g}$ vector encoding the flippase (pCAGGS-FLP, ref. 30) were co-electroporated with $1.5 \times 107 \mathrm{KH} 2 \mathrm{ES}$ cells at $400 \mathrm{~V}$ and $125 \mu \mathrm{F}$ using 2 pulses in a Gene Pulser II (Bio-Rad). Recombination between FRT sites such that the entire plasmid is inserted within the PGK promoter and the ATG initiation codon upstream and in frame with the hygromycin resistance gene was used to select correctly targeted cells. ES cells were treated with $140 \mu \mathrm{g} / \mathrm{mL}$ hygromycin B (Carl Roth) after 48 hours of electroporation to select clones that had undergone site-specific recombination and individual clones were picked after 8 to 14 days (31). Individual ES clones were tested in vitro by treating them with $1 \mu \mathrm{g} / \mathrm{mL}$ doxycycline (Sigma-Aldrich) in the culture media for 4 days. Then, GFP was measured in a FACSCanto II cytometer (BD Biosciences) to assess the electroporation efficiency, and cells expressing GFP were sorted.

Doxycycline induction. To induce CPT1A expression, 8-weekold Pax8-rtTA ${ }^{\text {tg } / 0}$ tetO-Cpt $1 \mathrm{a}^{\text {tg } / 0}$ mice and corresponding Pax8-rt$\mathrm{TA}^{\mathrm{o} / 0}$ tetO-Cpt1a ${ }^{\mathrm{tg} / 0}$ (WT) mice were fed with doxycycline (SigmaAldrich) at concentrations of $1 \mathrm{mg} / \mathrm{mL}$ via drinking water for 3 weeks. Mice were housed in colony cages with a 12-hour light/12hour dark cycle in a temperature- and humidity-controlled environment with free access to water.

Genotyping. Mice were genotyped by PCR using DNA extracted from tail biopsies and the primers listed in Supplemental Table 1. DNA extraction was performed by using the AccuStart II Mouse Genotyping Kit (Quantabio) according to the manufacturer's instructions.

Cell lines and culture conditions. Human proximal TECs (HKC-8) were cultured in DMEM/F12 1:1 (v/v) (Corning) supplemented with $15 \mathrm{mM}$ HEPES, 5\% (vol/vol) FBS (HyClone Laboratories), $1 \times$ insulin-transferrin-selenium (ITS) (Gibco), $0.5 \mu \mathrm{g} / \mathrm{mL}$ hydrocortisone (Sigma-Aldrich), 50 units $/ \mathrm{mL}$ penicillin, and $50 \mu \mathrm{g} / \mathrm{mL}$ streptomycin (Gibco). This cell line was provided in-house. HEK293A cells obtained from ATCC were cultured in DMEM supplemented with $10 \%(\mathrm{v} / \mathrm{v})$ FBS (HyClone Laboratories) and 1\% penicillin/streptomycin (Gibco). All cells were cultured at $37^{\circ} \mathrm{C}$ and $5 \% \mathrm{CO}_{2}$ and treated with trypsin every 5 days for subculturing. Treatments with human recombinant $10 \mathrm{ng} / \mathrm{mL}$ TGF- $\beta 1$ (R\&D Systems) were performed after serum-free starvation for 12 hours.

Isolation of primary kidney epithelial cells. Kidneys from CPT1A-KI and WT mice (3- to 5-week-old males) were collected after euthanization and minced into pieces of approximately $1 \mathrm{~mm}^{3}$. These pieces were digested with $10 \mathrm{~mL}$ HBSS containing $2 \mathrm{mg} / \mathrm{mL}$ collagenase I (Thermo Fisher Scientific) for 30 minutes at $37^{\circ} \mathrm{C}$ with gentle stirring, and supernatants were sieved through a $100 \mu \mathrm{m}$ nylon mesh. After centrifugation for 10 minutes at $300 \mathrm{~g}$, the pellet was resuspended in sterile red blood cell lysis buffer $\left(8.26 \mathrm{~g} \mathrm{NH}_{4} \mathrm{Cl}, 1 \mathrm{~g} \mathrm{KHCO}_{3}\right.$, and 0.037 
A

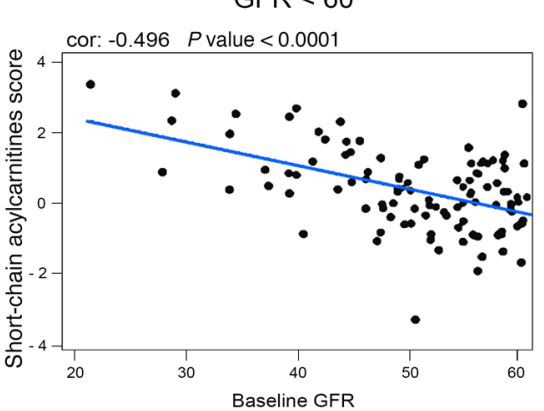

D

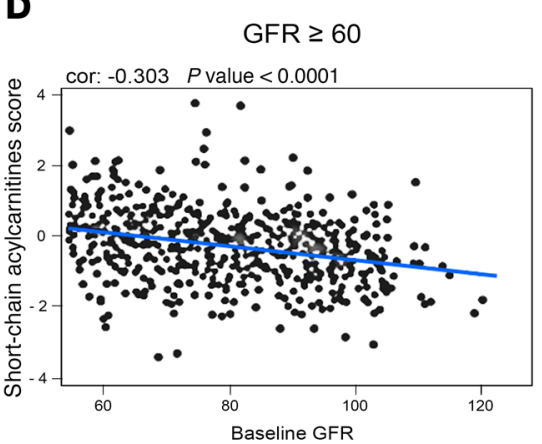

B

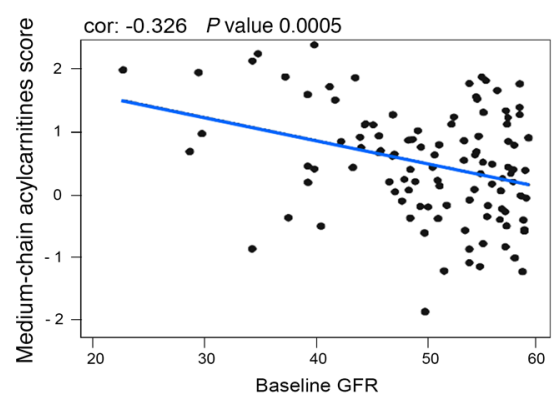

E

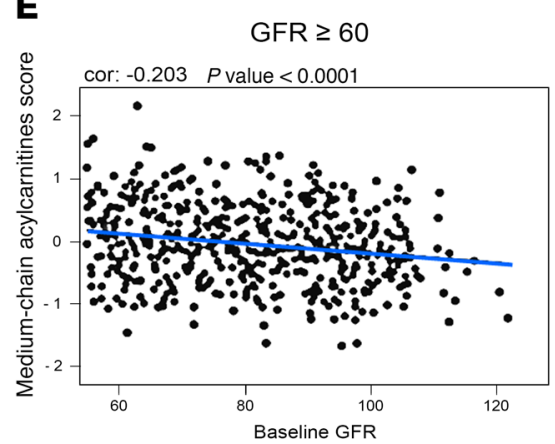

C

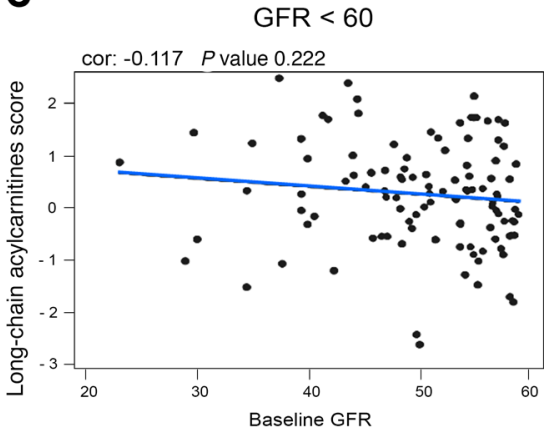

$\mathbf{F}$

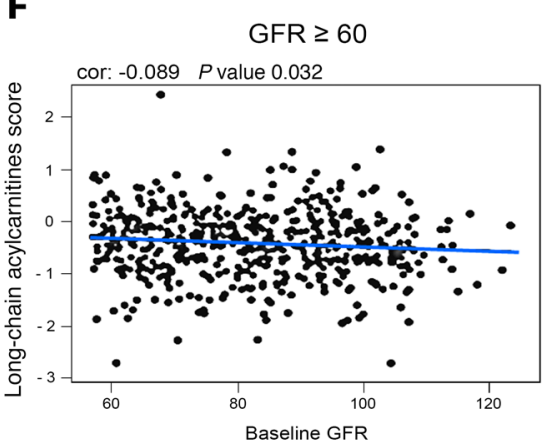

G

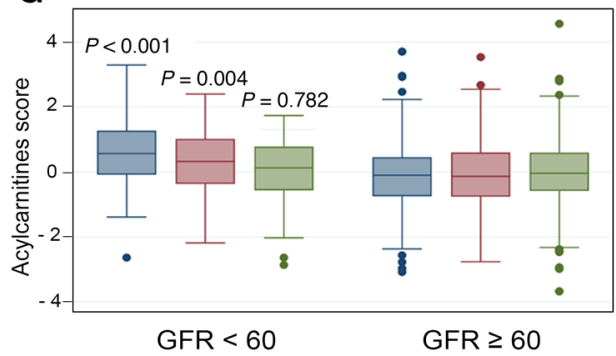

Short-chain acylcarnitines score

$\square$ Medium-chain acylcarnitines score Long-chain acylcarnitines score

H

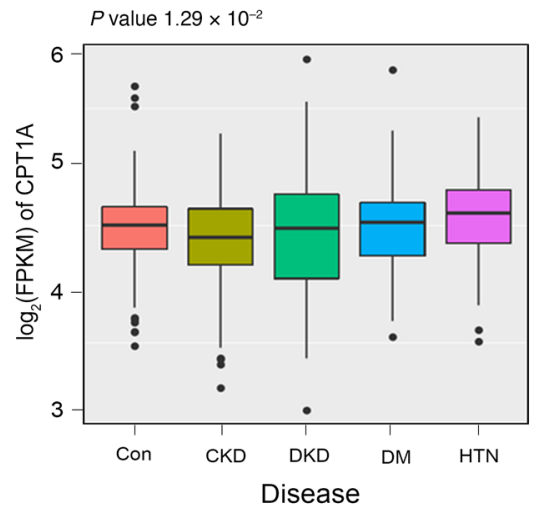

I

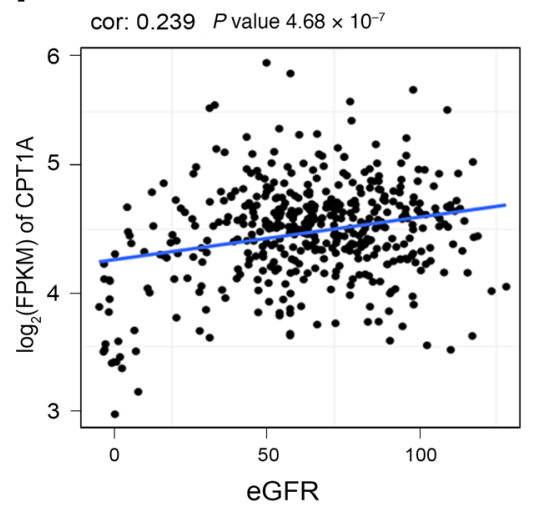

J

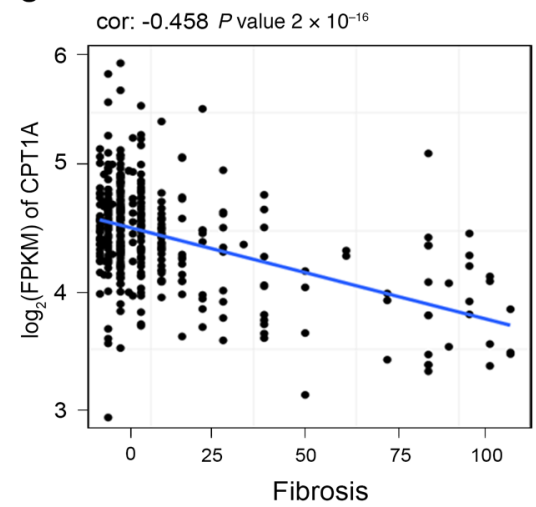

Figure 8. Plasma acylcarnitines and CPT1A levels in patients with CKD. (A-C) Correlation between baseline GFR values and plasma short-chain acylcarnitines (A), medium-chain acylcarnitines (B), and long-chain acylcarnitines (C) score in CKD patients with GFR less than $60 \mathrm{~mL} / \mathrm{min}$. (D-F) Correlation between baseline GFR values and plasma short-chain acylcarnitines (D), medium-chain acylcarnitines (E), and long-chain acylcarnitines (F) score in CKD patients with GFR greater than or equal to $60 \mathrm{~mL} / \mathrm{min}$. (G) Baseline acyl-carnitine levels by CKD stage. $P$ values for the comparison of acylcarnitine levels between participants with GFR lower than $60 \mathrm{~mL} / \mathrm{min}$ versus GFR greater than or equal to $60 \mathrm{~mL} / \mathrm{min}$. (H) CPT1A levels in renal biopsies from control and patients with CKD, diabetic kidney disease (DKD), diabetes mellitus (DM), or hypertension (HTN). (I and J) Correlation between CPT1A kidney levels and eGFR (I) or fibrosis score (J). For box-and-whisker plots $(\mathbf{G}$ and $\mathbf{H})$, within each box, horizontal white lines denote median values; boxes extend from the $25^{\text {th }}$ to the $75^{\text {th }}$ percentile of each group's distribution of values; vertical extending lines denote adjacent values (i.e., the most extreme values within 1.5 IQR of the 25th and 75th percentile of each group); dots denote observations outside the range of adjacent values. (A-G) $\chi^{2}$ and Student's $t$ test were used to compare categorical and quantitative variables, respectively. (H) ANOVA test was used to assess the significance across different disease groups. Cor.test function in $\mathrm{R}$ was used to obtain the Pearson's correlation and the corresponding $P$ values. 
g EDTA per $1 \mathrm{~L} \mathrm{ddH}_{2} \mathrm{O}$ ) and seeded in $10 \mathrm{~cm}$ culture dishes. Cells were cultured in RPMI 1640 (Corning) supplemented with 10\% FBS (HyClone Laboratories), $20 \mathrm{ng} / \mathrm{mL}$ EGF (Sigma-Aldrich), $20 \mathrm{ng} / \mathrm{mL}$ bFGF (Sigma-Aldrich), 50 units $/ \mathrm{mL}$ penicillin, and $50 \mu \mathrm{g} / \mathrm{mL}$ streptomycin (Gibco) at $37^{\circ} \mathrm{C}$ and $5 \% \mathrm{CO}_{2}$. Cells were used between days 7 and 10 of culture.

Immunoblot. Cells or a quarter piece of each kidney sample were homogenized and lysed in 100/300 $\mu \mathrm{L}$ RIPA lysis buffer containing $150 \mathrm{mM} \mathrm{NaCl}, 0.1 \%$ SDS, $1 \%$ sodium deoxycholate, $1 \% \mathrm{NP}-40$, and $25 \mathrm{mM}$ Tris- $\mathrm{HCl}$ pH 7.6 in the presence of cOmplete Protease Inhibitor Cocktail (Roche Diagnostics) and phosphatase inhibitor (SigmaAldrich). Samples were clarified by centrifugation at 10,000 $g$ for 15 minutes at $4^{\circ} \mathrm{C}$. Protein concentrations were determined by the BCA Protein Assay Kit (Thermo Fisher Scientific) and measured in GloMax-Multi Detection system (Promega). Equal amounts of protein $(10-50 \mu \mathrm{g})$ from the total extract were separated on $8 \%-10 \%$ SDS-polyacrylamide gels and transferred onto nitrocellulose blotting membranes (GE Healthcare) at $12 \mathrm{~V}$ for 20 minutes in a semidry TransBlot Turbo system (Bio-Rad). Membranes were blocked by incubation for 1 hour with 5\% nonfat milk in PBS containing 0.5\% Tween-20 and blotted overnight with the specific antibodies listed in Supplemental Table 2. After incubation with IRDye 800 goat anti-rabbit and IRDye 600 goat anti-mouse (1:15,000, LI-COR Biosciences) secondary antibodies, membranes were imaged with the Odyssey Infrared Imaging System (LI-COR Biosciences). Band densitometry was performed using Image 1.48 software (NIH), and relative protein expression was determined by normalizing to GAPDH. Fold changes were normalized to values of the control condition.

RNA extraction. Total RNA was extracted from HKC-8 or mouse kidneys using the miRNeasy Mini Kit (Qiagen) according to the manufacturer's instructions. RNA quantity and quality were determined at 260 $\mathrm{nm}$ by a Nanodrop-1000 spectrophotometer (Thermo Fisher Scientific).

Analysis of mRNA expression. Reverse transcription (RT) was carried out with $500 \mathrm{ng}$ of total RNA using the iScript cDNA Synthesis kit (Bio-Rad). qRT-PCR was carried out with the iQ SYBR Green Supermix (Bio-Rad), using a 96-well Bio-Rad CFX96 RT-PCR System with a C1000 Thermal Cycler according to the manufacturer's instructions. $\mathrm{A} \mathrm{C}_{\mathrm{t}}$ value was obtained from each amplification curve using CFX96 analysis software provided by the manufacturer. Relative mRNA expression was determined using the $2^{-\Delta \Delta \mathrm{Ct}}$ method (32). The $18 \mathrm{~S}$ gene was used for normalization purposes. The primer sequences used for mRNA quantification are listed in Supplemental Table 3. Fold changes were normalized to values of the control condition.

Mouse models of kidney fibrosis. Mice were housed in the specific pathogen-free animal facility at the CBMSO in accordance with EU regulations for all the procedures. UUO, FAN, and ADN models are described in Supplemental Methods.

$\left[1-{ }^{14} \mathrm{C}\right]$-palmitate oxidation. Measurement of FAO rates was performed in mouse kidney tissue and cells as previously described (33). Kidneys from renal fibrosis mouse models were homogenized in 5 volumes of chilled sodium chloride-Tris-EDTA buffer by a Dounce homogenizer. Next, $30 \mu \mathrm{L}$ of tissue homogenate supernatant was mixed with $370 \mu \mathrm{L}$ of the oxidation reaction mixture containing $7 \%$ BSA $/ 5 \mathrm{mM}$ palmitate $/ 0.01 \mu \mathrm{Ci} / \mu \mathrm{L}{ }^{14} \mathrm{C}$-palmitate (PerkinElmer) and incubated at $37^{\circ} \mathrm{C}$ for 30 minutes. Cells were seeded in 12-well dishes to reach a confluence of $70 \%$ and were infected with CPT1A adenoviruses as described in the adenovirus-mediated CPT1A overexpression procedure section. Then, cells were incubated in $500 \mu \mathrm{L}$ of media containing $0.3 \% \mathrm{BSA} / 100 \mu \mathrm{M}$ palmitate $/ 0.4 \mu \mathrm{Ci} / \mathrm{mL}^{14} \mathrm{C}$-palmitate at 37 ${ }^{\circ} \mathrm{C}$ for 3 hours. Each sample was assayed in triplicate. The reaction was stopped by the addition of $200 \mu \mathrm{L}$ of $1 \mathrm{M}$ perchloric acid. The rate of palmitate oxidation was measured as released ${ }^{14} \mathrm{CO}_{2}$ trapped in a filter paper disk with $20 \mu \mathrm{L}$ of $1 \mathrm{M} \mathrm{NaOH}$ in the top of sealed vials. The remaining acid solution for each sample was centrifuged at $14,000 \mathrm{~g}$ for 10 minutes at $4^{\circ} \mathrm{C}$ and $400 \mu \mathrm{L}$ of supernatant was added to scintillation vials for the quantification of ${ }^{14} \mathrm{C}$-palmitate-derived acid-soluble metabolites. ${ }^{14} \mathrm{C}$ products were counted in an LS6500 liquid scintillation counter (Beckman Coulter). Scintillation values were converted to $\mathrm{mmol}{ }^{14} \mathrm{CO}_{2}$ or acid-soluble metabolites by multiplying the specific activity and normalized to the protein content.

TaqMan gene expression assay. TaqMan array plates (Thermo Fisher Scientific) were selected as the platform for gene expression profiling. This panel consisted of a total of 43 unique TaqMan probes specific for mouse genes related to fibrosis, apoptosis, mitochondrial metabolism, glucose utilization, and inflammation listed in Supplemental Table 4. RNA extraction from kidney samples was performed as described in the RNA extraction procedure section. Next, $2 \mu \mathrm{g}$ of total RNA was subjected to reverse transcription using the High-Capacity cDNA Reverse Transcription Kit (Thermo Fisher Scientific) according to the manufacturer's instructions. PCR amplification was performed using TaqMan master (Thermo Fisher Scientific) with the Roche LightCycler 480 Real-Time PCR system (AB7900HT). This procedure was performed in the Genomic Facility of the Fundación Parque Científico de Madrid. The ABI TaqMan SDS v2.4 software was utilized to obtain Cq values (Cq) for each gene. The Cq data were analyzed with StatMiner 4.2.8 software (Integromics). The $\Delta \mathrm{C}_{\mathrm{t}}$ value was calculated by normalizing $C_{t}$ values to the endogenous housekeeping gene 18S. Relative mRNA expression was determined using the $2^{-\Delta \Delta \mathrm{Ct}}$ method (32). Fold changes were normalized to values of the control condition.

Mitochondrial copy number determination. Genomic DNA was extracted from mouse kidneys using the DNeasy Blood \& Tissue Kit (Qiagen) according to the manufacturer's instructions. Mitochondrial abundance was determined with the Mouse Mitochondrial DNA Copy Number Assay Kit (Detroit R\&D). Relative mtDNA copy number was presented as the mtDNA to nuclear DNA ratio.

Assessment of kidney function. Animal kidney function was determined by analyzing the indicators serum creatinine and BUN by using the QuantiChrom TM Creatinine Assay Kit (BioAssay Systems) and the BUN Colorimetric Detection Kit (Arbor Assays), respectively, according to the manufacturers' instructions. In the case of the reversion experiments (Figure 7), serum creatinine was measured by capillary electrophoresis (Agilent 7100) coupled to TOF mass spectrometry (Agilent 6224) $(34,35)$. A calibration curve was used for quantification with creatinine; methionine sulphone was the internal standard.

Measurement of ATP level. ATP content in kidney tissue, HKC-8, and primary kidney epithelial cells was measured by using the ATP colorimetric/fluorometric assay kit (BioVision Inc.) according to the manufacturer's instructions. Data were normalized for total protein content. In HKC-8 cells, relative ATP content was also assessed with the FRET Clover-mApple ATP sensor $(36,37)$. Cells were seeded in 12-well dishes to reach a confluence of $70 \%$ and transfected with 1.5 $\mu \mathrm{g}$ vector/well using Lipofectamine 2000 (Invitrogen). Then, cells were infected with CPT1A adenoviruses as described in the adenovirus-mediated CPT1A overexpression procedure section and FRET 
signal was analyzed by flow cytometry. Cells were gated by forward (FSC-A) and side scatter (SSC-A) and for single cells using FSC-A/ FSC-H. Next, dead cells were excluded. Fluorescence intensity was measured by flow cytometry using wavelengths (ex/em) 550/610 (acceptor), 490/550 (donor), and 490/600 (FRET) in a FACSCanto II system (BD Biosciences) and analyzed with FlowJo 10.2 software. The ratio of FRET/donor was displayed as a histogram and its median of fluorescence intensity was obtained. For each experimental condition, at least 20,000 singlets were analyzed in triplicates.

Quantification of kidney cell populations by flow cytometry. Multiparametric flow cytometry was used for the identification of the macrophage and epithelial cell population. Kidneys were diced, incubated at $37^{\circ} \mathrm{C}$ for 30 minutes with $0.5 \mathrm{mg} / \mathrm{mL}$ Liberase DL (Roche Basel) and $100 \mathrm{U} / \mathrm{mL}$ DNase (Roche Basel) in serum-free DMEM, and filtered $(40 \mu \mathrm{m})$. For staining, $10^{6}$ cells were dissolved in $50 \mu \mathrm{L}$ of buffer and preincubated with $0.25 \mu \mathrm{L}$ CD16/CD32 (BioLegend). Next, $3 \mu \mathrm{L}$ of DAPI (1:15000) (Sigma-Aldrich) was added to stain dead cells. Cell suspensions were incubated with specific fluorochromeconjugated antibodies (BioLegend) listed in Supplemental Table 5. For each experiment, flow minus one (FMO) controls were performed for each fluorophore to establish gates by using corresponding antibodies listed in Supplemental Table 5. Fluorescence intensity was measured in a FACSCanto II cytometer (BD Biosciences) and analyzed with FlowJo 10.2 software. For each kidney sample, at least 20,000 singlets were analyzed in triplicates. Cell gating was initially based in the forward versus side scatter (FSC vs. SSC) plot. Next, dead cells were excluded. Identification of the macrophage cell population was based on the presence of CD45, expressed in inflammatory and hematopoietic cells and F4/80, a specific macrophage surface marker. CD86 and CD206 were used to determine M1 and M2 macrophage subpopulations, respectively (38). Identification of the epithelial cell population was based on the presence of the epithelial cell adhesion molecule (EpCAM) and the absence of CD45. The subsequent display of positive CD24 determined injured proximal tubule epithelia (39). Numbers in quadrants indicate cell proportions in the percentage of cells that coexpress both markers.

Measurements of OCR. FAO-associated OCR (ligated to oxidative phosphorylation) and ECAR (associated with lactate production and glycolysis) were studied using the Seahorse Bioscience metabolic analyzer for the XF Cell Mito Stress Test and Agilent Seahorse XF RealTime ATP Rate Assay according to the manufacturer's instructions (40). See Supplemental Methods for details.

Immunofluorescence. A quarter piece of each kidney sample was immersed sequentially in $4 \%$ neutral buffered formalin for 24 hours, in $30 \%$ sucrose in PBS until tissue sank (6-12 hours), and embedded in Tissue-Tek OCT for cryoprotection at $-80^{\circ} \mathrm{C}$. OCT blocks were cut in serial frozen sections (10 $\mu \mathrm{m}$ thickness). These sections were fixed with $4 \%$ PFA for 10 minutes and permeabilized with $0.25 \%$ Triton $\mathrm{X}-100$ in PBS for 5 minutes at room temperature (RT). Next, they were blocked with 1\% BSA in PBS for 30 minutes at RT and incubated for 1 hour for staining with specific primary antibodies and fluorochrome-conjugated secondary antibodies for 1 hour at RT (Supplemental Table 6). Nuclei were stained with DAPI (Sigma) for 5 minutes at RT. The coverslips were mounted on slides using MOWIOL (Calbiochem). Tissue fluorescence was visualized by an LSM 510 Meta Confocal microscope with a $40 \times / 1.3$ oil Plan-Neofluar M27 objective (Zeiss).

Histology and immunohistochemistry. A quarter piece of each kidney sample was immersed in $4 \%$ neutral buffered formalin for 24 hours, embedded in paraffin, cut in serial sections (5 $\mu \mathrm{m}$ thickness), and stained with H\&E, Sirius red, and periodic acid-Schiff (PAS) as described previously (41) Tissue sections for immunostaining were deparaffinized through xylene and hydrated through graded ethanol (100\%, 96\%, 90\%, and 70\%) and distilled water. Endogenous peroxidase was blocked. We incubated the tissue sections with $4 \%$ BSA for 1 hour at RT. Primary antibodies to detect CD3 (M7254, 1:100, Dako) were incubated for 30 minutes at RT. After washing, slides were incubated with goat anti-mouse HRP secondary antibody ("Visualization Reagent HRP" from SK006, Dako) for 30 minutes and visualized using the PD-L1 IHC 22C3 pharmDx (SK006, Dako). To detect Kim-1, primary antibody (AF1817, 1:500, R\&D Systems) was incubated for 30 minutes at RT. To detect F4/80, primary antibody (MCA4976, 1:50, BioRad) was incubated at $4^{\circ} \mathrm{C}$ overnight. After washing, slides were incubated with goat anti-rabbit IgG biotin-SP conjugate secondary antibody (31752, 1:200, Invitrogen) and rabbit anti-rat IgG biotin-SP (31834, 1:200, Thermo Fisher Scientific), respectively, and visualized using DAB Substrate Kit (ab64238, Abcam). Tissue sections were revealed using DAB $(20 \mu \mathrm{L} / \mathrm{mL}$, Dako) and counterstained with Carazzi's hematoxylin. Slides were mounted with MOWIOL (Calbiochem). See Supplemental Methods for histological and immunohistochemical analysis.

Electron microscopy examination. The number of mitochondria and their structure was analyzed by standard transmission electron microscopy. Renal cortex pieces were cut into small blocks $\left(1 \mathrm{~mm}^{3}\right)$ and fixed by immersion in fixative (4\% paraformaldehyde/ $2 \%$ glutaraldehyde in $0.1 \mathrm{M}$ phosphate buffer) overnight at $4^{\circ} \mathrm{C}$. After washing with cold PBS, they were incubated in $1 \%$ osmium tetroxide and $1 \%$ potassium ferricyanide in water for 1 hour at $4^{\circ} \mathrm{C}$. The pieces were sequentially stained with $0.15 \%$ tannic acid (in $0.1 \mathrm{M}$ phosphate buffer) for 1 minute at RT and 2\% uranyl acetate in water for 1 hour at RT. Next, they were dehydrated in graded ethanol and embedded in EmBed812 resin (Electron Microscopy Sciences). Serial ultrathin sections $(70 \mathrm{~nm})$ of the tissue were collected on copper mesh grids. Three grids from each sample were examined using a JEOL 1230 transmission electron microscope, and digital photographs were captured by real-time digital imaging.

Adenovirus-mediated CPT1A overexpression. CPT1A overexpression in cells was driven and transduced by adenoviral particles. Adenoviruses carrying CPT1A (AdCPT1A) or adenovirus control (AdControl) were amplified, purified, and titrated according to Calderon-Dominguez et al. (ref. 42, see Supplemental Methods for details). HCK8 cells were seeded into $60 \mathrm{~mm}$ culture dishes to reach a confluence of $70 \%$. They were infected with adenoviruses AdControl and AdCPT1A (100 MOI) for 24 hours in serum-free DMEM/ F12 (supplemented with $15 \mathrm{mM}$ HEPES, 5\% FBS, $1 \times$ ITS, $0.5 \mu \mathrm{g} / \mathrm{mL}$ hydrocortisone, 50 units $/ \mathrm{mL}$ penicillin, and $50 \mu \mathrm{g} / \mathrm{mL}$ streptomycin), and then the medium was replaced with complete medium for an additional 24 hours. Adenovirus infection efficiency was assessed in AdControl-infected cells by immunofluorescence.

Clinical data. Acylcarnitines were evaluated based on the PREDIMED trial. The PREDIMED is a primary prevention, multicenter trial conducted in Spain. A detailed description of this trial is provided elsewhere (13). Liquid chromatography-tandem mass spectrometry was used to semiquantitatively profile acylcarnitines in plasma samples. Our analysis is based on a subsample of 686 participants with metabolomics data and measures of kidney function, including GFR and urinary albu- 
min-creatinine ratio $(43,44)$. Studies of CPT1A expression in kidney tubules were performed in a cohort of 433 patients described in Beckerman et al. (45) and herein named the CKD cohort, whose demographic and clinical features are summarized in Supplemental Table 9.

See complete unedited blots in the supplemental material.

Statistics. Data in experimental models were analyzed using nonparametric tests except where indicated. The difference between 2 independent groups was examined with the Mann-Whitney $U$ test; more than 2 groups were compared with the Kruskal-Wallis test. A $P$ value of 0.05 or less was considered statistically significant. Data were analyzed using GraphPad Prism 6.0. Data are reported as mean \pm SEM. See Supplemental Materials for statistics of data in clinical studies for acylcarnitines and CPT1A expression.

Study approval. Animals were handled in agreement with the Guide for the Care and Use of Laboratory Animals contained in Directive 2010/63/EU of the European Parliament. Approval was granted by the CBMSO Ethics Committee for Animal Experimentation of the CBMSO in Madrid, the ethics committee from Consejo Superior de Investigaciones Científicas (CSIC), and the Regulatory Unit for Animal Experimental Procedures from the Comunidad de Madrid. For human studies, written informed consent of participants is specified in the references $(44,45)$.

\section{Author contributions}

SL conceived and directed research. VM designed, performed, and analyzed the majority of experiments. JT and LME performed experiments. JIH provided technical assistance for mouse experiments. CC performed histological evaluation. LH and DS provided the CPT1A adenoviruses. MRO, DRP, PC, and CB provided intellectual and technical insight. RR assisted with the TaqMan analysis. SO coordinated the generation of the CPT1A-KI mouse model. XS, KS, MRC, JSS, and MAMG performed studies in 2 cohorts of CKD patients. All authors helped with the discussion of the results, and SL and VM wrote the manuscript.

\section{Acknowledgments}

This work was supported by grants from the Ministerio de Economía y Competitividad (MINECO) SAF2012-31388 (SL)，SAF2015-66107-R (SL)，PID2019-104233RB-100 (SL), PI17/01513 (DRP), SAF2017-83813-C3-1-R (DS and LH), cofunded by the European Regional Development Fund, Instituto de Salud Carlos III REDinREN RD12/0021/0009 and RD16/0009/0016 (SL, DRP, and MRO), PI17/00119 (MRO), FIS PI17/00625 (DRP), Comunidad de Madrid "NOVELREN" B2017/BMD-3751 (SL, DRP, and MRO), a grant-in-aid from the
Spanish Society of Nephrology (Fundación Senefro 2017) (SL) and Fundación Renal "Iñigo Alvarez de Toledo" (SL), the Centro de Investigación Biomédica en Red de Fisiopatología de la Obesidad y la Nutrición (CIBEROBN) CB06/03/0001 (DS), the Government of Catalonia 2017SGR278 (DS), and the Fundació La Marató de TV3 201627-30 (DS), all from Spain. The CBMSO receives institutional support from Fundación "Ramón Areces." The Prevención con Dieta Mediterránea (PREDIMED) trial was supported by grants from MINECO; the Instituto de Salud Carlos III RTIC G03/140; the Centro de Investigación Biomédica en Red de Fisiopatología de la Obesidad y Nutrición; the Centro Nacional de Investigaciones Cardiovasculares CNIC 06/2007; the Fondo de Investigación Sanitaria Fondo Europeo de Desarrollo Regional PIO4-2239, PI 05/2584, CP06/00100, PIO7/0240, PI07/1138, PI07/0954, PI 07/0473, PI10/01407, PI10/02658, PI11/01647, P11/02505, and PI13/00462; the Ministerio de Ciencia e Innovación AGL-2009-13906-C02 and AGL2010-22319-C03; the Fundación Mapfre 2010; Consejería de Salud de la Junta de Andalucía PI0105/2007; the Public Health Division of the Department of Health of the Autonomous Government of Catalonia, Generalitat Valenciana ACOMP06109, GVA-COMP2010-181, GVACOMP2011-151, CS2010-AP-111, and CS2011-AP-042; and the Regional Government of Navarra P27/2011. Metabolomics measurements in PREDIMED were funded by NIH R01DK102896, F31DK114938, NIH/NHLBI 1R01HL118264, and NIH/NHLBI 2R01HL118264. PREDIMED funding is related to MRC, MA-MG, and JS. VM was supported by predoctoral fellowships of the Formación de Personal Investigador (FPI) program (BES-2013065986) from MINECO. We acknowledge the J. David Gladstone Institutes and Ken Nakamura (University of California) for providing the Clover-mApple ATP sensor, Miguel López (Universidad de Santiago de Compostela) for providing the p-AMPK and p-ACC antibodies, Maria Luisa Toribio (CBMSO) for her assistance with the flow cytometry analysis, and Germán Andrés (CBMSO) for his help with electron microscopy image evaluation. We are grateful to the laboratories of Jorgina Satrústegui and José Manuel Cuezva for sharing the Seahorse equipment and Laura Santos for her help with histology procedures, from the laboratory of Marta Ruiz-Ortega at the Fundación Jiménez Díaz. We also thank the following facilities of the CBMSO: animal housing, flow cytometry, and confocal and electron microscopy.

Address correspondence to: Santiago Lamas, Centro de Biología Molecular "Severo Ochoa" (CSIC-UAM), Nicolás Cabrera 1, 28049 Madrid, Spain.Phone: 34.91.196.4455; Email: slamas@cbm.csic.es.
1. Kazancioglu R. Risk factors for chronic kidney disease: an update. Kidney Int Suppl (2011). 2013;3(4):368-371.

2. Drawz PE, Rosenberg ME. Slowing progression of chronic kidney disease. Kidney Int Suppl (2011). 2013;3(4):372-376

3. Kang HM, et al. Defective fatty acid oxidation in renal tubular epithelial cells has a key role in kidney fibrosis development. Nat Med. 2015;21(1):37-46.

4. Chung KW, et al. Mitochondrial damage and activation of the STING pathway lead to renal inflammation and fibrosis. Cell Metab. 2019;30(4):784-799.

5. Ly JP, et al. Mouse models to study kidney development, function and disease. Curr Opin Nephrol Hypertens. 2011;20(4):382-390.

6. Stadler K, et al. The evolving understanding of the contribution of lipid metabolism to diabetic kidney disease. Curr Diab Rep. 2015;15(7):40.

7. Eirin A, et al. The emerging role of mitochondrial targeting in kidney disease. Handb Exp Pharmacol. 2017;240:229-250.

8. Mihaylova MM, Shaw RJ. The AMPK signalling pathway coordinates cell growth, autophagy and metabolism. Nat Cell Biol. 2011;13(9):1016-1023.

9. Imig JD, Ryan MJ. Immune and inflammatory role in renal disease. Compr Physiol. 2013;3(2):957-976.

10. Kusaba T, et al. Differentiated kidney epithelial cells repair injured proximal tubule. Proc Natl Acad Sci U S A. 2014;111(4):1527-1532.

11. Grgic I, et al. The origin of interstitial myofibroblasts in chronic kidney disease. Pediatr Nephrol. 2012;27(2):183-193.

12. Huang S, Susztak K. Epithelial plasticity ver- 
sus EMT in kidney fibrosis. Trends Mol Med. 2016;22(1):4-6.

13. Estruch R, et al. Primary prevention of cardiovascular disease with a Mediterranean diet supplemented with extra-virgin olive oil or nuts. N Engl J Med. 2018;378(25):e34.

14. Duann P, Lin PH. Mitochondria damage and kidney disease. Adv Exp Med Biol. 2017;982:529-551.

15. Schlaepfer IR, Joshi M. CPT1A-mediated fat oxidation, mechanisms, and therapeutic potential. Endocrinology. 2020;161(2):bqz046.

16. Nyman LR, et al. Homozygous carnitine palmitoyltransferase 1a (liver isoform) deficiency is lethal in the mouse. Mol Genet Metab. 2005;86(1-2):179-187.

17. Schoors $\mathrm{S}$, et al. Fatty acid carbon is essential for dNTP synthesis in endothelial cells. Nature. 2015;520(7546):192-197.

18. Briant LJB, et al. CPT1a-dependent long-chain fatty acid oxidation contributes to maintaining glucagon secretion from pancreatic islets. Cell Rep. 2018;23(11):3300-3311.

19. Mihaylova MM, et al. Fasting activates fatty acid oxidation to enhance intestinal stem cell function during homeostasis and aging. Cell Stem Cell. 2018;22(5):769-778.

20. Matthaei KI. Genetically manipulated mice: a powerful tool with unsuspected caveats. JPhysiol. 2007;582(Pt 2):481-488.

21. Afshinnia $\mathrm{F}$, et al. Impaired $\beta$-oxidation and altered complex lipid fatty acid partitioning with advancing CKD.JAm Soc Nephrol. 2018;29(1):295-306.

22. Adams SH, et al. Plasma acylcarnitine profiles suggest incomplete long-chain fatty acid betaoxidation and altered tricarboxylic acid cycle activity in type 2 diabetic African-American women. J Nutr. 2009;139(6):1073-1081.

23. Schooneman MG, et al. Transorgan fluxes in a porcine model reveal a central role for liver in acylcarnitine metabolism. Am J Physiol Endocrinol Metab. 2015;309(3):E256-E264.

24. Miyamoto Y, et al. Retention of acetylcarnitine in chronic kidney disease causes insulin resistance in skeletal muscle. JClin Biochem Nutr. 2016;59(3):199-206.

25. Hue L, Taegtmeyer H. The Randle cycle revisited: a new head for an old hat. Am J Physiol Endocrinol Metab. 2009;297(3):E578-E591.

26. Mather A, Pollock C. Glucose handling by the kidney. Kidney Int Suppl. 2011;(120):S1-S6.

27. Hochedlinger K, et al. Ectopic expression of Oct- 4 blocks progenitor-cell differentiation and causes dysplasia in epithelial tissues. Cell. 2005;121(3):465-477.

28. Beard C, et al. Efficient method to generate single-copy transgenic mice by site-specific integration in embryonic stem cells. Genesis. 2006;44(1):23-28.

29. Kuzmichev AN, et al. Sox 2 acts through Sox 21 to regulate transcription in pluripotent and differentiated cells. Curr Biol. 2012;22(18):1705-1710.

30. Buchholz F, et al. Improved properties of FLP recombinase evolved by cycling mutagenesis. Nat Biotechnol.1998;16(7):657-662.

31. Kumar RM, et al. Deconstructing transcriptional heterogeneity in pluripotent stem cells. Nature. 2014;516(7529):56-61.

32. Livak KJ, Schmittgen TD. Analysis of relative gene expression data using real-time quantitative PCR and the 2(-Delta Delta C(T)) Method. Methods. 2001;25(4):402-408.

33. Huynh FK, et al. Measurement of fatty acid oxidation rates in animal tissues and cell lines. Methods Enzymol. 2014;542:391-405.

34. Harada S, et al. Reliability of plasma polar metabolite concentrations in a large-scale cohort study using capillary electrophoresis-mass spectrometry. PLoS One. 2018;13(1):e0191230.

35. Hirayama A, et al. Metabolic profiling reveals new serum biomarkers for differentiating diabetic nephropathy. Anal Bioanal Chem. 2012;404(10):3101-3109.

36. Imamura $H$, et al. Visualization of ATP levels inside single living cells with fluorescence resonance energy transfer-based genetically encoded indicators. Proc Natl Acad Sci U S A. 2009;106(37):15651-15656.

37. Mendelsohn BA, et al. A high-throughput screen of real-time ATP levels in individual cells reveals mechanisms of energy failure. PLoS Biol. 2018;16(8):e2004624.

38. Misharin AV, et al. Flow cytometric analysis of macrophages and dendritic cell subsets in the mouse lung. Am JRespir Cell Mol Biol. 2013;49(4):503-510.

39. Smeets B, et al. Proximal tubular cells contain a phenotypically distinct, scattered cell population involved in tubular regeneration. J Pathol. 2013;229(5):645-659.

40. van der Windt GJ, et al. Measuring bioenergetics in T cells using a seahorse extracellular flux analyzer. Curr Protoc Immunol. 2016;113:3.16B.1-3.16B.14.

41. Varga J, Brenner D, Phan S, eds. Fibrosis Research -Methods and Protocols. Humana Press; 2005.

42. Calderon-Dominguez M, et al. Carnitine palmitoyltransferase 1 increases lipolysis, UCP1 protein expression and mitochondrial activity in brown adipocytes. PLoS One. 2016;11(7):e0159399.

43. Diaz-Lopez A, et al. Cross-sectional associations between macronutrient intake and chronic kidney disease in a population at high cardiovascular risk. Clin Nutr. 2013;32(4):606-612.

44. Guasch-Ferre M, et al. Plasma acylcarnitines and risk of type 2 diabetes in a Mediterranean population at high cardiovascular risk. JClin Endocrinol Metab. 2019;104(5):1508-1519.

45. Beckerman P, et al. Human kidney tubule-specific gene expression based dissection of chronic kidney disease traits. EBioMedicine. 2017;24:267-276. 